\title{
Article \\ Robust Aggregation Operators for Intuitionistic Fuzzy Hypersoft Set with Their Application to Solve MCDM Problem
}

\author{
Rana Muhammad Zulqarnain ${ }^{1, *(\mathbb{D})}$, Imran Siddique ${ }^{2, *}$, Rifaqat Ali ${ }^{3}$, Dragan Pamucar ${ }^{4}$ (D), Dragan Marinkovic ${ }^{5}$ \\ and Darko Bozanic ${ }^{6}$ (i)
}

1 Department of Mathematics, University of Management and Technology, Sialkot Campus, Lahore 51310, Pakistan

2 Department of Mathematics, University of Management and Technology, Lahore 54770, Pakistan

3 Department of Mathematics, College of Science and Arts, Muhayil, King Khalid University, Abha 61413, Saudi Arabia; rrafat@kku.edu.sa

4 Department of Logistics, Military academy, University of Defence in Belgarde, Pavla Jurisica Sturma 33, 11000 Belgrade, Serbia; dragan.pamucar@va.mod.gov.rs

5 Faculty of Mechanical and Transport Systems, Technische Universität Berlin, 10623 Berlin, Germany; dragan.marinkovic@tu-berlin.de

6 Military Academy, University of Defence in Belgarde, Pavla Jurisica Sturma 33, 11000 Belgrade, Serbia; dbozanic@yahoo.com

* Correspondence: muhammad.zulqarnain@skt.umt.edu.pk (R.M.Z.); imransiddique@umt.edu.pk (I.S.)

check for updates

Citation: Zulqarnain, R.M.; Siddique, I.; Ali, R.; Pamucar, D.; Marinkovic, D.; Bozanic, D. Robust Aggregation Operators for Intuitionistic Fuzzy Hypersoft Set with Their Application to Solve MCDM Problem. Entropy 2021, 23, 688. https://doi.org/10.3390/ e23060688

Academic Editor: Jaesung Lee

Received: 22 April 2021

Accepted: 26 May 2021

Published: 29 May 2021

Publisher's Note: MDPI stays neutral with regard to jurisdictional claims in published maps and institutional affiliations.

Copyright: (c) 2021 by the authors. Licensee MDPI, Basel, Switzerland. This article is an open access article distributed under the terms and conditions of the Creative Commons Attribution (CC BY) license (https:// creativecommons.org/licenses/by/ $4.0 /)$.

\begin{abstract}
In this paper, we investigate the multi-criteria decision-making complications under intuitionistic fuzzy hypersoft set (IFHSS) information. The IFHSS is a proper extension of the intuitionistic fuzzy soft set (IFSS) which discusses the parametrization of multi-sub attributes of considered parameters, and accommodates more hesitation comparative to IFSS utilizing the multi sub-attributes of the considered parameters. The main objective of this research is to introduce operational laws for intuitionistic fuzzy hypersoft numbers (IFHSNs). Additionally, based on developed operational laws two aggregation operators (AOs), i.e., intuitionistic fuzzy hypersoft weighted average (IFHSWA) and intuitionistic fuzzy hypersoft weighted geometric (IFHSWG), operators have been presented with their fundamental properties. Furthermore, a decision-making approach has been established utilizing our developed aggregation operators (AOs). Through the established approach, a technique for solving decision-making (DM) complications is proposed to select sustainable suppliers in sustainable supply chain management (SSCM). Moreover, a numerical description is presented to ensure the validity and usability of the proposed technique in the DM process. The practicality, effectivity, and flexibility of the current approach are demonstrated through comparative analysis with the assistance of some prevailing studies.
\end{abstract}

Keywords: hypersoft set; intuitionistic fuzzy soft set; intuitionistic fuzzy hypersoft set; IFHSWA operator; IFHSWG operator; SSCM

\section{Introduction}

Decision-making is an interesting concern to select the perfect alternative for any particular purpose. Firstly, it is supposed that details about alternatives are accumulated in crisp numbers, but in real-life situations, collective farm information always contains wrong and inaccurate information. Fuzzy sets [1] are similar to sets having an element of membership (Mem) degree. In classical set theory, the Mem degree of the elements in a set is examined in binary form to see that the element is not entirely concomitant to the set. In contrast, the fuzzy set theory enables advanced Mem categorization of the components in the set. It is portrayed by the Mem function, and also the multipurpose unit interval of the Mem function is [0, 1]. In some circumstances, decision-makers consider the Mem and non-membership (Nmem) values of objects. In such cases Zadeh's FS is unable to handle the imprecise and vague information. Atanassov [2] established the notion of 
intuitionistic fuzzy sets (IFS) to deal with the above-mentioned concerns. In addition, several other theories have been proposed to overcome such complications, such as cubic IFS [3], interval-valued IFS [4], entropy and distance measures for IFSS [5], etc.

Atanassov's IFS accommodates the inadequate information utilizing Mem and NMem values, but IFS is unable to deal with incompatible and inexact data in which alternatives have parametric values. To overcome such complications, Molodtsov [6] presented the soft set (SS) for indeterminate, uncertain, and vague substances. Maji et al. [7] established the notion of the fuzzy soft set (FSS) by merging FS and SS. Ali et al. [8] proposed a novel decision-making approach for bipolar FSS utilizing different types of parameter reduction. Maji et al. [9] developed the notion of IFSS and presented some fundamental operations with their desirable properties. Garg and Arora [10] introduced the correlation coefficient for IFSS, and extended the TOPSIS method for IFSS. They also utilized their developed TOPSIS technique to solve multi-attribute decision-making (MADM) obstacles. Wei and Zhang [11] presented two novel entropy measures for IFS and interval-valued IFS based on cosine function with their fundamental properties and established a decision-making technique to utilize their developed entropy measures. Zulqarnain et al. [12] proposed the correlation coefficient for IVFSS and developed the TOPSIS technique, utilizing their presented correlation coefficient. They also introduced the AOs for IVFSS, and used their established techniques to solve decision-making complications. Garg and Arora [13] extended the notion of IFSS, proposed the most generalized form of IFSS, and developed some AOs with their fundamental properties.

Wang et al. [14] extended the PFSs and introduced interactive Hamacher operations with some novel AOs. They also established a DM method to solve MADM problems by using their proposed operators. Wang and Li [15] extended the notion of PFSs to intervalvalued PFSs with some desirable operators. They also developed a DM technique utilizing their proposed operators to solve multi-attribute group decision-making (MAGDM) problems. Wang et al. [16] established a MADM approach utilizing interval-valued q-rung orthopair 2-tuple linguistic. Pamucar [17] established an MCDM approach by merging two existing studies of interval grey numbers and normalized weighted geometric DombiBonferroni mean operators. Peng and Yuan [18] established some novel operators, such as Pythagorean fuzzy point operators, and developed a DM technique using their proposed operators. Garg [19] extended the weighted AOs to PFSs and acquired numerous operators, introducing a DM technique founded on settled operators. Wang and Liu [20] studied a intuitionistic fuzzy Einstein weighted geometric operator and established a DM approach to solving DM complications. Garg [21] developed the logarithmic operational laws for PFSs and proposed some AOs. Wang and Liu [22] introduced the intuitionistic fuzzy Einstein weighted averaging operator and the intuitionistic fuzzy Einstein ordered weighted averaging operator with their desirable properties. Arora and Garg [23] presented the operational laws for linguistic IFS and developed prioritized AOs.

Faizi et al. [24] introduced two novel techniques to obtain the best priority vector for the solution of MAGDM problems utilizing intuitionist 2-tuple linguistic sets. Based on Hamacher operations, they presented some operational laws in an under-considered environment. $\mathrm{Xu}$ [25] presented some innovative AOs for IFSs and proposed the comparison laws for IFNs. Sinani et al. [26] extended the Hamy mean and Dombi operators for rough numbers and established a DM approach to resolving MADM challenges based on their acquired operators. Riaz et al. [27] introduced the soft multi-set topology on a soft multi-set with aggregation operators and the MCDM technique. Peng et al. [28] presented the notion of PFSS with its fundamental properties merging two prevailing techniques, PFS and SS. Athira et al. [29] extended the concept of PFSS, and introduced entropy measure under-considered environment. Zulqarnain et al. [30] established operational laws for Pythagorean fuzzy soft numbers (PFSNs) and developed AOs, such as Pythagorean fuzzy soft weighted average and geometric, by using defined operational laws for PFSNs. They also planned a DM approach to solve MADM problems with the help of presented operators. Riaz et al. [31] have established AOs utilizing Einstein Operations and examined 
their fundamental properties; they also proposed a DM technique to solve MCDM obstacles. Faizi et al. [32] extended the notion of normalized interval-valued triangular fuzzy numbers, and developed an MCDM technique to solve DM issues. Several techniques have been established to solve multi-criteria decision analysis (MCDA) which provide suitable results in real-life complications [33,34]. Salabun et al. [35] worked to benchmark selected MCDA techniques and determined that a set of realistic MCDA approaches. Referring to the mentioned guide, they propose to organize simulation experimentations.

The existing studies only deal with inadequate information due to membership and non-membership values. However, these theories cannot handle the overall incompatible, as well as imprecise, information. When any attribute from a set of parameters comprises further sub-attributes, the prevailing theories fail to solve such types of problems. To overcome the aforementioned limitations, Smarandache [36] developed the idea of SS to hypersoft sets (HSS) by substituting the one-parameter function $\mathrm{f}$ to a multi-parameter (sub-attribute) function. Samarandache claimed that the established HSS can competently deal with uncertain objects, in comparison to SS. Nowadays, HSS theory and its extensions have been gaining unexpected traction. Several investigators have examined progressed distinctive operators along with characteristics under HSS and its extensions [37-43]. Zulqarnain et al. [44] presented the IFHSS, which is the generalized version of IFSS. They established the TOPSIS method to resolve the MADM problem, utilizing the developed correlation coefficient.

The essential objective of the following scientific research is to grow novel AOs for the IFHSS environment and processing mechanism, which can also follow the assumptions of IFHSNs. Furthermore, we developed an algorithm to explain the MCDM problem, and presented a numerical illustration to justify the effectiveness of the proposed approach under the IFHSS environment. Supplier selection and evaluation is a critical factor in business activity. Recent government policy changes have led to supplier selection being considered from various perspectives, including environmental and social imperatives. Thus, in the literature, the problem is referred to as sustainable supplier selection and described as a problem for MCDM. Simultaneously, many papers [45-48] point to the need for further research using MCDM methods in sustainable supplier selection, oriented at properly reflecting uncertainties in the data of the environment and the preferences of the decision-maker. To improve the computing power and flexibility of IFSS, we first summarize the decision formula to incorporate the views of decision-makers into IFHSS terms, and propose operational rules for IFHSS. According to the newly developed operational rules, there are two AOs, namely IFHSWA and IFHSWG operators, which have been established. Many of the associated properties of these operators are also inspected. The score function and accuracy function of IHFSS are also discussed to compare IFHSS. The algorithmic rule following the proposed operator to resolve the DM problems is anticipated, along with a numerical example used to demonstrate the effectiveness of the introduced DM approach.

The rest of the study is planned thus: In Section 2, we present some initial impressions, such as SS, HSS, and IFHSS, which help us to build a successful investigation structure. We formulated some operational laws for IFHSNs in accordance with established operating laws, and presented the aggregation operators (AOs) with their properties in Section 3. In Section 4, a DM method is developed for SSCM by using the proposed operator. To ensure the practicality of the established DM approach a numerical example is presented. In addition, we utilize some available techniques to indicate analysis within our own designed technique. Furthermore, we give the benefits of planned algorithms, simplicity, flexibility, and effectivity. In Section 5, we will concisely discuss and compare existing techniques along with the projected approach. 


\section{Preliminaries}

In the following section, we are going to review some fundamental definitions that support us in establishing the following research structures, such as SS, HSS, FHSS, as well as IFHSS.

Definition 1. [6] Let $\mathcal{U}$ be the universal set and $\mathcal{E}$ be the set of attributes concerning $\mathcal{U}$. Let $\mathcal{P}(\mathcal{U})$ be the power set of $\mathcal{U}$ and $C \subseteq \mathcal{E}$. A pair $(\mathcal{F}, C)$ is called a $S S$ over $\mathcal{U}$ and its mapping is given as

$$
\mathcal{F}: C \rightarrow \mathcal{P}(\mathcal{U})
$$

It is also defined as:

$$
(\mathcal{F}, C)=\{\mathcal{F}(e) \in \mathcal{P}(\mathcal{U}): e \in \mathcal{E}, \mathcal{F}(e)=\varnothing \text { if } e \notin C\}
$$

Definition 2. [7] $\mathcal{F}(\mathcal{U})$ be a collection of all fuzzy subsets over $\mathcal{U}$ and $\mathcal{E}$ be a set of attributes. Let $C \subseteq \mathcal{E}$, then a pair $(\mathcal{F}, C)$ is called FSS over $\mathcal{U}$, where $\mathcal{F}$ is a mapping such as

$$
\mathcal{F}: C \rightarrow \mathcal{F}(\mathcal{U})
$$

Definition 3. [36] Let $\mathcal{U}$ be a universe of discourse and $\mathcal{P}(\mathcal{U})$ be a power set of $\mathcal{U}$ and $k=\left\{k_{1}, k_{2}, k_{3}, \ldots, k_{n}\right\},(n \geq 1)$ be a set of attributes and set $K_{i}$ a set of corresponding sub-attributes of $k_{i}$ respectively with $K_{i} \cap K_{j}=\varphi$ for $n \geq 1$ for each $i, j \varepsilon\{1,2,3 \ldots n\}$ and $i \neq j$. Assume $K_{1} \times K_{2} \times K_{3} \times \ldots \times K_{n}=\ddot{C}=\left\{c_{1 h} \times c_{2 k} \times, \ldots, \times c_{n l}\right\}$ be a collection of multi-attributes, where $1 \leq h \leq \alpha, 1 \leq k \leq \beta$, and $1 \leq l \leq \gamma$, and $\alpha, \beta$, and $\gamma \in \mathbb{N}$. Then the pair $\left(\mathcal{F}, K_{1} \times K_{2} \times\right.$ $\left.K_{3} \times \ldots \times K_{n}=\dddot{C}\right)$ is said to be HSS over $\mathcal{U}$ and its mapping is defined as

$$
\mathcal{F}: K_{1} \times K_{2} \times K_{3} \times \ldots \times K_{n}=\dddot{C} \rightarrow \mathcal{P}(\mathcal{U})
$$

It is also defined as

$$
(\mathcal{F}, \dddot{C})=\left\{(\check{a}, \mathcal{F} \dddot{a}(\check{a})): \check{a} \in \dddot{A}, \mathcal{F}_{\dddot{A}}(\check{a}) \in \mathcal{P}(\mathcal{U})\right\}
$$

Definition 4. [44] Let $\mathcal{U}$ be a universe of discourse and $\mathcal{P}(\mathcal{U})$ be a power set of $\mathcal{U}$ and $k=\left\{k_{1}, k_{2}, k_{3}, \ldots, k_{n}\right\},(n \geq 1)$ be a set of attributes and set $K_{i}$ a set of corresponding subattributes of $k_{i}$ respectively with $K_{i} \cap K_{j}=\varphi$ for $n \geq 1$ for each $i, j \varepsilon\{1,2,3 \ldots n\}$ and $i \neq j$. Assume $K_{1} \times K_{2} \times K_{3} \times \ldots \times K_{n}=\dddot{C}=\left\{c_{1 h} \times c_{2 k} \times \cdots \times c_{n l}\right\}$ be a collection of sub-attributes, where $1 \leq h \leq \alpha, 1 \leq k \leq \beta$, and $1 \leq l \leq \gamma$, and $\alpha, \beta$, and $\gamma \in \mathbb{N}$ and IFS $\mathcal{H}^{\mathcal{U}}$ be collection of all intuitionistic fuzzy subsets over $\mathcal{U}$. Then the pair $\left(\mathcal{F}, K_{1} \times K_{2} \times K_{3} \times \ldots \times K_{n}=\dddot{C}\right)$ is said to be IFHSS over $\mathcal{U}$, and its mapping is defined as

$$
\mathcal{F}: K_{1} \times K_{2} \times K_{3} \times \ldots \times K_{n} \rightarrow I F S^{\mathcal{U}} .
$$

It is also defined as

$$
(\mathcal{F}, \dddot{C})=\left\{\left(\check{c}, \mathcal{F}_{\dddot{C}}(\check{c})\right): \check{c} \in \dddot{C}, \mathcal{F}_{\dddot{C}}(\check{c}) \in I F S^{\mathcal{U}} \in[0,1]\right\}
$$

where $\mathcal{F}_{\dddot{C}}(\check{c})=\left\{\delta, \mu_{\mathcal{F}(\check{c})}(\delta), \vartheta_{\mathcal{F}(\check{c})}(\delta): \delta \in \mathcal{U}\right\}$, where $\mu_{\mathcal{F}(\check{c})}(\delta)$ and $\vartheta_{\mathcal{F}(\check{c})}(\delta)$ represents the $M D$ and NMD of the sub-attributes of the considered parameters such as $\mu_{\mathcal{F}(\check{c})}(\delta)$, $\vartheta_{\mathcal{F}(\breve{c})}(\delta) \in[0,1]$, and $0 \leq \mu_{\mathcal{F}(\breve{c})}(\delta)+\vartheta_{\mathcal{F}(\breve{c})}(\delta) \leq 1$. 
Remark 1. If $\mu_{\mathcal{F}(\check{c})}(\delta)+\vartheta_{\mathcal{F}(\check{c})}(\delta) \leq 1$ is held and all parameters of a set of attributes have no further sub-attribute. Then, IFHSS was reduced to IFSS [9].

The IFHSN $\mathcal{F}_{\delta_{i}}\left(\check{c}_{j}\right)=\left\{\left(\mu_{\mathcal{F}\left(\check{c}_{j}\right)}\left(\delta_{i}\right), \vartheta_{\mathcal{F}\left(\check{c}_{j}\right)}\left(\delta_{i}\right)\right) \mid \delta_{i} \in \mathcal{U}\right\}$ can be express as $\mathfrak{J}_{\check{c}_{i j}}=\left\langle\mu_{\mathcal{F}\left(\check{c}_{i j}\right)}, \vartheta_{\mathcal{F}\left(\check{c}_{i j}\right)}\right\rangle$ for readers' convenience. To rank the alternatives scoring function of $\mathfrak{J}_{i j}$ is defined in the following:

$$
\mathbb{S}\left(\mathfrak{J}_{\check{c}_{i j}}\right)=\mu_{\mathcal{F}\left(\check{c}_{i j}\right)}-\vartheta_{\mathcal{F}\left(c_{i j}\right)}, \mathbb{S}\left(\mathfrak{J}_{\check{c}_{i j}}\right) \in[-1,1]
$$

However, sometimes the scoring function such as $\mathfrak{J}_{\check{c}_{11}}=\langle 0.4,0.7\rangle$ and $\mathfrak{J}_{\check{c}_{12}}=\langle 0.5,0.8\rangle$ is unable to compute the two IFHSNs. In such cases it can be difficult to decide which value is most suitable $\mathbb{S}\left(\mathfrak{J}_{\check{c}_{11}}\right)=0.3=\mathbb{S}\left(\mathfrak{J}_{\check{c}_{12}}\right)$. Accuracy function has been introduced to overcome such difficulties:

$$
\mathrm{H}\left(\mathfrak{J}_{\check{c}_{i j}}\right)=\mu_{\mathcal{F}\left(\check{c}_{i j}\right)}+\vartheta_{\mathcal{F}\left(\check{c}_{i j}\right)}, \mathrm{H}\left(\mathfrak{J}_{\check{c}_{i j}}\right) \in[0,1] .
$$

Thus, to compare two IFHSNs $\mathfrak{J}_{\check{c}_{i j}}$ and $\mathfrak{T}_{\check{c}_{i j}}$, the subsequent ranking and comparison laws are classified as follows:

1. If $\mathbb{S}\left(\mathfrak{J}_{\check{c}_{i j}}\right)>\mathbb{S}\left(\mathfrak{T}_{c_{i j}}\right)$, then $\mathfrak{J}_{\check{c}_{i j}}>\mathfrak{T}_{\check{c}_{i j}}$.

2. If $\mathbb{S}\left(\mathfrak{J}_{\check{c}_{i j}}\right)=\mathbb{S}\left(\mathfrak{T}_{\check{c}_{i j}}\right)$, then

o If $\mathrm{H}\left(\mathfrak{J}_{\check{c}_{i j}}\right)>\mathrm{H}\left(\mathfrak{T}_{\check{c}_{i j}}\right)$, then $\mathfrak{J}_{\check{c}_{i j}}>\mathfrak{T}_{\check{c}_{i j}}$

o If $\mathrm{H}\left(\mathfrak{J}_{\check{c}_{i j}}\right)=\mathrm{H}\left(\mathfrak{T}_{\check{c}_{i j}}\right)$, then $\mathfrak{J}_{\check{c}_{i j}}=\mathfrak{T}_{\check{c}_{i j}}$.

\section{Aggregation Operators for Intuitionistic Fuzzy Hypersoft Numbers}

In this section, we present the operational laws for IFHSNs and propose the IFHSWA, and IFHSWG operators for IFHSS. Furthermore, we discuss the fundamental properties of IFHSWA and IFHSWG operators utilizing our developed IFHSNs.

\subsection{Operational Laws for Intuitionistic Fuzzy Hypersoft Numbers}

Definition 5. Let $\mathfrak{J}_{\check{c}_{k}}=\left(\mu_{\check{c}_{k}}, \vartheta_{\check{c}_{k}}\right), \mathfrak{J}_{\check{c}_{11}}=\left(\mu_{\check{c}_{11}}, \vartheta_{\check{c}_{11}}\right)$, and $\mathfrak{J}_{\check{c}_{12}}=\left(\mu_{\check{c}_{12}}, \vartheta_{\check{c}_{12}}\right)$ be three IFHSNs and $\alpha$ be a positive real number, by algebraic norms, we have

1. $\mathfrak{J}_{\check{c}_{11}} \oplus \mathfrak{J}_{\check{c}_{12}}=\left\langle\mu_{\check{c}_{11}}+\mu_{\check{c}_{12}}-\mu_{\check{c}_{11}} \mu_{\check{c}_{12}}, \vartheta_{\check{c}_{11}} \vartheta_{\check{c}_{12}}\right\rangle$

2. $\mathfrak{J}_{\check{c}_{11}} \otimes \mathfrak{J}_{c_{12}}=\left\langle\mu_{\check{c}_{11}} \mu_{\check{c}_{12}}, \vartheta_{\check{c}_{11}}+\vartheta_{\check{c}_{12}}-\vartheta_{\check{c}_{11}} \vartheta_{\check{c}_{12}}\right\rangle$

3. $\alpha \mathfrak{J}_{\check{c}_{k}}=\left\langle\left[1-\left(1-\mu_{\check{c}_{k}}\right)^{\alpha},\left(\vartheta_{\check{c}_{k}}\right)^{\alpha}\right]\right\rangle$

4. $\quad \mathfrak{J}_{\check{c}_{k}}^{\alpha}=\left\langle\left[\left(\mu_{\check{c}_{k}}\right)^{\alpha}, 1-\left(1-\vartheta_{\check{c}_{k}}\right)^{\alpha}\right]\right\rangle$

Some average and geometric AOs for IFHSSs are described based on the above rules for collecting IFHSNs $\Delta$.

Definition 6. Let $\mathfrak{J}_{\check{c}_{k}}=\left(\mu_{\check{c}_{k}}, \vartheta_{\check{c}_{k}}\right)$ be an IFHSN, $\Omega_{i}$ and $\gamma_{j}$ are weight vector for expert's and sub-attributes of selected parameters respectively with given conditions $\Omega_{i}>0, \sum_{i=1}^{n} \Omega_{i}=1$, $\gamma_{j}>0, \sum_{j=1}^{m} \gamma_{j}=1$. Then IFHSWA operator is defined as

IFHSWA: $\Delta^{n} \rightarrow \Delta$ defined as follows:

$$
\operatorname{SIFHSWA}\left(\mathfrak{J}_{\check{c}_{11}}, \mathfrak{J}_{\check{c}_{12}}, \ldots, \mathfrak{J}_{\check{c}_{n m}}\right)=\oplus_{j=1}^{m} \gamma_{j}\left(\oplus_{i=1}^{n} \Omega_{i} \mathfrak{J}_{\check{c}_{i j}}\right) .
$$


Theorem 1. Let $\mathfrak{J}_{\check{c}_{k}}=\left(\mu_{\check{c}_{k}}, \vartheta_{\check{c}_{k}}\right)$ be an IFHSN. Then, the aggregated values obtained by using Equation (3) is also an IFHSN and

$$
=\left\langle\begin{array}{c}
\text { IFHSWA }\left(\mathfrak{J}_{\check{c}_{11}}, \mathfrak{J}_{\check{c}_{12}}, \ldots, \mathfrak{J}_{\check{c}_{n m}}\right) \\
=\left\langle 1-\prod_{j=1}^{m}\left(\prod_{i=1}^{n}\left(1-\mu_{\check{c}_{i j}}\right)^{\Omega_{i}}\right)^{\gamma_{j}}, \prod_{j=1}^{m}\left(\prod_{i=1}^{n}\left(\vartheta_{\check{c}_{i j}}\right)^{\Omega_{i}}\right)^{\gamma_{j}}\right\rangle .
\end{array}\right.
$$

where $\Omega_{i}$ and $\gamma_{j}$ are weight vector for expert's and sub-attributes of the parameters correspondingly with given circumstances $\Omega_{i}>0, \sum_{i=1}^{n} \Omega_{i}=1, \gamma_{j}>0, \sum_{j=1}^{m} \gamma_{j}=1$.

Proof. We can prove this by applying the principle of mathematical induction such as follows:

For $n=1$, we get $\Omega_{1}=1$. Then, we have

$$
\begin{aligned}
& \operatorname{IFHSWA}\left(\mathfrak{J}_{\check{c}_{11}}, \mathfrak{J}_{\check{c}_{12}}, \ldots, \mathfrak{J}_{\check{c}_{n m}}\right)=\oplus^{m}{ }_{j=1} \gamma_{j} \mathfrak{J}_{\check{c}_{i j}} \\
& \operatorname{IFHSWA}\left(\mathfrak{J}_{\check{c}_{11}}, \mathfrak{J}_{\check{c}_{12}}, \ldots, \mathfrak{J}_{\check{c}_{n m}}\right)=\left\langle 1-\prod_{j=1}^{m}\left(1-\mu_{\check{c}_{1 j}}{ }^{2}\right)^{\gamma_{j}}, \prod_{j=1}^{m}\left(\vartheta_{\check{c}_{1 j}}\right)^{\gamma_{j}}\right\rangle \\
& =\left\langle 1-\prod_{j=1}^{m}\left(\prod_{i=1}^{1}\left(1-\mu_{\check{d}_{1 j}}{ }^{2}\right)^{\Omega_{i}}\right)^{\gamma_{j}}, \prod_{j=1}^{m}\left(\prod_{i=1}^{1}\left(\vartheta_{\check{d}_{1 j}}\right)^{\Omega_{i}}\right)^{\gamma_{j}}\right\rangle .
\end{aligned}
$$

For $m=1$, we get $\gamma_{1}=1$. Then, we have

$$
\begin{aligned}
& \text { IFHSWA }\left(\mathfrak{J}_{\check{c}_{11}}, \mathfrak{J}_{\check{c}_{12}}, \ldots, \mathfrak{J}_{\check{c}_{n m}}\right)=\oplus_{i=1}^{n} \Omega_{i} \mathfrak{J}_{\check{c}_{i j}} \\
& =\left\langle 1-\prod_{i=1}^{n}\left(1-\mu_{\check{c}_{i 1}}{ }^{2}\right)^{\Omega_{i}}, \prod_{i=1}^{n}\left(\vartheta_{\check{c}_{i 1}}\right)^{\Omega_{i}}\right\rangle \\
& =\left\langle 1-\prod_{j=1}^{1}\left(\prod_{i=1}^{n}\left(1-\mu_{\check{c}_{i j}}{ }^{2}\right)^{\Omega_{i}}\right)^{\gamma_{j}}, \prod_{j=1}^{1}\left(\prod_{i=1}^{n}\left(\vartheta_{\check{c}_{i j}}\right)^{\Omega_{i}}\right)^{\gamma_{j}}\right\rangle .
\end{aligned}
$$

This shows that Equation (4) satisfies for $n=1$ and $m=1$. Consider Equation (4) holds for $m=\beta_{1}+1, n=\beta_{2}$ and $m=\beta_{1}, n=\beta_{2}+1$, such as:

$$
\begin{aligned}
& \oplus_{j=1}^{\beta_{1}+1} \gamma_{j}\left(\oplus_{i=1}^{\beta_{2}} \Omega_{i} \mathfrak{J}_{\check{c}_{i j}}\right)=\left\langle 1-\prod_{j=1}^{\beta_{1}+1}\left(\prod_{i=1}^{\beta_{2}}\left(1-\mu_{\check{c}_{i j}}{ }^{2}\right)^{\Omega_{i}}\right)^{\gamma_{j}}, \prod_{j=1}^{\beta_{1}+1}\left(\prod_{i=1}^{\beta_{2}}\left(\vartheta_{\check{c}_{i j}}\right)^{\Omega_{i}}\right)^{\gamma_{j}}\right\rangle \\
& \oplus_{j=1}^{\beta_{1}} \gamma_{j}\left(\oplus_{i=1}^{\beta_{2}+1} \Omega_{i} \mathfrak{J}_{\check{c}_{i j}}\right)=\left\langle 1-\prod_{j=1}^{\beta_{1}}\left(\prod_{i=1}^{\beta_{2}+1}\left(1-\mu_{\check{c}_{i j}}{ }^{2}\right)^{\Omega_{i}}\right)^{\gamma_{j}}, \prod_{j=1}^{\beta_{1}}\left(\prod_{i=1}^{\beta_{2}+1}\left(\vartheta_{\check{c}_{i j}}\right)^{\Omega_{i}}\right)^{\gamma_{j}}\right\rangle
\end{aligned}
$$

For $m=\beta_{1}+1$ and $n=\beta_{2}+1$, we have

$$
\begin{aligned}
& \oplus_{j=1}^{\beta_{1}+1} \gamma_{j}\left(\oplus_{i=1}^{\beta_{2}+1} \Omega_{i} \mathfrak{J}_{c_{i j}}\right)=\oplus_{j=1}^{\beta_{1}+1} \gamma_{j}\left(\oplus_{i=1}^{\beta_{2}} \Omega_{i} \mathfrak{J}_{\check{c}_{i j}} \oplus \Omega_{\beta_{2}+1} \mathfrak{J}_{\left(\beta_{2}+1\right) j}\right) \\
& =\oplus_{j=1}^{\beta_{1}+1} \oplus_{i=1}^{\beta_{2}} \gamma_{j} \Omega_{i} \mathfrak{J}_{i j} \oplus_{j=1}^{\beta_{1}+1} \gamma_{j} \Omega_{\beta_{2}+1} \mathfrak{J}_{\check{c}_{\left(\beta_{2}+1\right) j}} \\
& =\left\langle\begin{array}{c}
1-\prod_{j=1}^{\beta_{1}+1}\left(\prod_{i=1}^{\beta_{2}}\left(1-\mu_{\check{c}_{i j}}{ }^{2}\right)^{\Omega_{i}}\right)^{\gamma_{j}} \oplus 1-\prod_{j=1}^{\beta_{1}+1}\left(\left(1-\mu_{\check{c}_{\left(\beta_{2}+1\right) j}}{ }^{2}\right)^{\Omega_{\beta_{2}+1}}\right)^{\gamma_{j}}, \\
\prod_{j=1}^{\beta_{1}+1}\left(\prod_{i=1}^{\beta_{2}}\left(\vartheta_{\check{c}_{i j}}\right)^{\Omega_{i}}\right)^{\gamma_{j}} \oplus \prod_{j=1}^{\beta_{1}+1}\left(\left(\vartheta_{\check{c}_{\left(\beta_{2}+1\right) j}}\right)^{\Omega_{\beta_{2}+1}}\right)^{\gamma_{j}}
\end{array}\right\rangle \\
& =\left\langle 1-\prod_{j=1}^{\beta_{1}+1}\left(\prod_{i=1}^{\beta_{2}+1}\left(1-\mu_{\check{c}_{i j}}{ }^{2}\right)^{\Omega_{i}}\right)^{\gamma_{j}}, \Pi_{j=1}^{\beta_{1}+1}\left(\prod_{i=1}^{\beta_{2}+1}\left(\vartheta_{\check{c}_{i j}}\right)^{\Omega_{i}}\right)^{\gamma_{j}}\right\rangle \text {. }
\end{aligned}
$$

Hence, it is true for $m=\beta_{1}+1$ and $n=\beta_{2}+1$.

\subsection{Properties of IFHSWA Operator}

3.2.1. (Idempotency)

If $\mathfrak{J}_{\check{c}_{i j}}=\mathfrak{J}_{\check{c}_{i j}}=\left(\mu_{\check{c}_{i j}}, \vartheta_{\check{c}_{i j}}\right) \forall i, j$, then, 
$\operatorname{IFHSWA}\left(\mathfrak{J}_{\check{c}_{11}}, \mathfrak{J}_{\check{c}_{12}}, \ldots, \mathfrak{J}_{\check{c}_{n m}}\right)=\mathfrak{J}_{\check{c}}$.

Proof. As we know $\mathfrak{J}_{\check{c}_{i j}}=\mathfrak{J}_{\check{c}}=\left(\mu_{\check{c}_{i j}} \vartheta_{\check{c}_{i j}}\right)$ to be a collection of IFHSNs, then by using Equation (4).

$$
\begin{aligned}
& \text { IFHSWA }\left(\mathfrak{J}_{\check{c}_{11}}, \mathfrak{J}_{\check{c}_{12}}, \ldots, \mathfrak{J}_{\check{c}_{n m}}\right)=\left\langle 1-\prod_{j=1}^{m}\left(\prod_{i=1}^{n}\left(1-\mu_{\check{c}_{i j}}\right)^{\Omega_{i}}\right)^{\gamma_{j}}, \prod_{j=1}^{m}\left(\prod_{i=1}^{n}\left(\vartheta_{\check{c}_{i j}}\right)^{\Omega_{i}}\right)^{\gamma_{j}}\right\rangle \\
& =\left\langle 1-\left(\left(1-\mu_{\check{c}_{i j}}\right)^{\sum_{i=1}^{n} \Omega_{i}}\right)^{\sum_{j=1}^{m} \gamma_{j}},\left(\left(\vartheta_{\check{c}_{i j}}\right)^{\sum_{i=1}^{n} \Omega_{i}}\right)^{\sum_{j=1}^{m} \gamma_{j}}\right\rangle \\
& =\left\langle 1-\left(1-\mu_{\check{c}_{i j}}\right), \vartheta_{\check{c}_{i j}}\right\rangle=\left(\mu_{\check{c}_{i j}}, \vartheta_{\check{c}_{i j}}\right)=\mathfrak{J}_{\check{c}} .
\end{aligned}
$$

Which completes the proof.

\subsection{2. (Boundedness)}

Let $\mathfrak{J}_{\check{c}_{i j}}$ be a collection of IFHSNs and

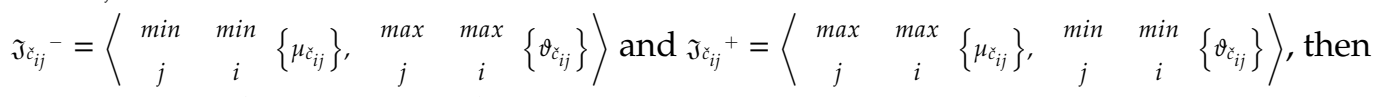
$\mathfrak{J}_{\tilde{c}_{i j}}{ }^{-} \leq \operatorname{IFHSWA}\left(\mathfrak{J}_{\tilde{c}_{11}}, \mathfrak{J}_{\tilde{c}_{12}}, \ldots, \mathfrak{J}_{\tilde{c}_{n m}}\right) \leq \mathfrak{J}_{\tilde{c}_{i j}}{ }^{+}$.

Proof. As we know $\mathfrak{J}_{\check{c}_{i j}}=\left(\mu_{\check{c}_{i j}}, \mathcal{J}_{\check{c}_{i j}}\right)$ to be an IFHSN, then

$$
\begin{aligned}
& \min _{j} \min _{i}\left\{\mu_{\check{c}_{i j}}\right\} \leq \mu_{\check{c}_{i j}} \leq \underset{j}{\max } \max _{i}\left\{\mu_{\check{c}_{i j}}\right\} \\
& \Rightarrow 1-\underset{j}{\max } \max _{i}\left\{\mu_{\check{c}_{i j}}\right\} \leq 1-\mu_{\check{c}_{i j}} \leq 1-\min _{j} \min _{i}\left\{\mu_{\check{c}_{i j}}\right\} \\
& \Leftrightarrow\left(1-\max _{j} \max _{i}\left\{\mu_{\check{c}_{i j}}\right\}\right)^{\Omega_{i}} \leq\left(1-\mu_{\check{c}_{i j}}\right)^{\Omega_{i}} \leq\left(1-\min _{j} \min _{i}\left\{\mu_{\check{c}_{i j}}\right\}\right)^{\Omega_{i}} \\
& \Leftrightarrow\left(1-\max _{j} \max _{i}\left\{\mu_{\check{c}_{i j}}\right\}\right)^{\sum_{i=1}^{n} \Omega_{i}} \leq \prod_{i=1}^{n}\left(1-\mu_{\check{c}_{i j}}\right)^{\Omega_{i}} \leq\left(1-\underset{j}{\min } \min _{i}\left\{\mu_{\check{c}_{i j}}\right\}\right)^{\sum_{i=1}^{n} \Omega_{i}} \\
& \Leftrightarrow\left(1-\max _{j} \max _{i}\left\{\mu_{\check{c}_{i j}}\right\}\right)^{\sum_{j=1}^{m} \gamma_{j}} \leq \prod_{j=1}^{m}\left(\prod_{i=1}^{n}\left(1-\mu_{\check{c}_{i j}}\right)^{\Omega_{i}}\right)^{\gamma_{j}} \leq\left(1-\underset{j}{\min } \min _{i}\left\{\mu_{\check{c}_{i j}}\right\}\right)^{\sum_{j=1}^{m} \gamma_{j}} \\
& \Leftrightarrow 1-\underset{j}{\max } \max _{i}\left\{\mu_{\check{\mu}_{i j}}\right\} \leq \prod_{j=1}^{m}\left(\prod_{i=1}^{n}\left(1-\mu_{\check{c}_{i j}}\right)^{\Omega_{i}}\right)^{\gamma_{j}} \leq 1-\underset{j}{\min } \min _{i}\left\{\mu_{\check{c}_{i j}}\right\} \\
& \Leftrightarrow \min _{j} \min _{i}\left\{\mu_{\check{c}_{i j}}\right\} \leq 1-\prod_{j=1}^{m}\left(\prod_{i=1}^{n}\left(1-\mu_{\check{c}_{i j}}\right)^{\Omega_{i}}\right)^{\gamma_{j}} \leq \underset{j}{\max } \underset{i}{\max }\left\{\mu_{\check{c}_{i j}}\right\} \\
& \Leftrightarrow \min _{j} \min _{i}\left\{\mu_{\check{c}_{i j}}\right\} \leq 1-\prod_{j=1}^{m}\left(\prod_{i=1}^{n}\left(1-\mu_{\check{c}_{i j}}\right)^{\Omega_{i}}\right)^{\gamma_{j}} \leq \underset{j}{\max } \max _{i}\left\{\mu_{\check{c}_{i j}}\right\}
\end{aligned}
$$

Similarly,

$$
\min _{j} \min _{i}\left\{\vartheta_{\check{c}_{i j}}\right\} \leq \prod_{j=1}^{m}\left(\prod_{i=1}^{n}\left(\vartheta_{\check{c}_{i j}}\right)^{\Omega_{i}}\right)^{\gamma_{j}} \leq \underset{j}{\max } \max _{i}\left\{\vartheta_{\check{c}_{i j}}\right\}
$$

Let IFHSWA $\left(\mathfrak{J}_{\check{c}_{11}}, \mathfrak{J}_{\check{c}_{12}}, \ldots, \mathfrak{J}_{\check{c}_{n m}}\right)=\mu_{\check{c}_{\delta}}, \vartheta_{\check{c}_{\delta}}=\mathfrak{J}_{\check{c}_{\delta}}$, that inequities 5 and 6 could be turned into the subsequent form:

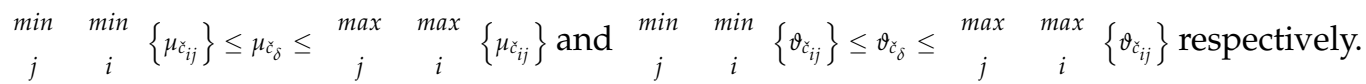

So, by utilizing Equation (1), we have:

$$
\mathbb{S}\left(\mathfrak{J}_{\check{c}_{\delta}}\right)=\mu_{\check{c}_{\delta}}-\vartheta_{\check{c}_{\delta}} \leq \underset{j}{\max } \max _{i}\left\{\mu_{\check{c}_{i j}}\right\}-\underset{j}{\min } \min \left\{\vartheta_{\check{c}_{i j}}\right\}=\mathbb{S}\left(\mathfrak{J}_{\check{c}_{i j}}{ }^{+}\right),
$$


$\mathbb{S}\left(\mathfrak{J}_{\check{c}_{\delta}}\right)=\mu_{\check{c}_{\delta}}-\vartheta_{\check{c}_{\delta}} \geq \min _{j} \min _{i}\left\{\mu_{\check{c}_{\delta}}\right\}-\max _{j} \max _{i}\left\{\vartheta_{\check{c}_{\delta}}\right\}=\mathbb{S}\left(\mathfrak{J}_{\check{c}_{i j}}{ }^{-}\right)$. Then, by order relation among two IFHSNs, we have

$\mathfrak{J}_{\check{c}_{i j}}{ }^{-} \leq \operatorname{IFHSWA}\left(\mathfrak{J}_{\check{c}_{11}}, \mathfrak{J}_{\check{c}_{12}}, \ldots, \mathfrak{J}_{\check{c}_{n m}}\right) \leq \mathfrak{J}_{\check{c}_{i j}}{ }^{+}$

3.2.3. (Shift Invariance) If $\mathfrak{J}_{\check{c}_{\delta}}=\left\langle\mu_{\check{c}_{\delta}}, \vartheta_{\check{c}_{\delta}}\right\rangle$ Be an IFHSN. Then,

$\operatorname{IFHSWA}\left(\mathfrak{J}_{c_{11}} \oplus \mathfrak{J}_{\check{c}_{\delta}}, \mathfrak{J}_{\check{c}_{12}} \oplus \mathfrak{J}_{\check{c}_{\delta}}, \ldots, \mathfrak{J}_{\check{c}_{n m}} \oplus \mathfrak{J}_{\check{c}_{\delta}}\right)=\operatorname{IFHSWA}\left(\mathfrak{J}_{\check{c}_{11}}, \mathfrak{J}_{\check{c}_{12}}, \ldots, \mathfrak{J}_{\check{c}_{n m}}\right) \oplus \mathfrak{J}_{\check{c}_{\delta}}$.

Proof. Consider $\mathfrak{J}_{\check{c}_{\delta}}$ and $\mathfrak{J}_{\check{c}_{i j}}$ to be two IFHSNs. Then, by using operational laws defined under IFHSNs in Definition 5 (1), we have:

$\mathfrak{J}_{\check{c}_{\delta}} \oplus \mathfrak{J}_{c_{i j}}=\left\langle\mu_{\check{c}_{\delta}}+\mu_{\check{c}_{i j}}-\mu_{\check{c}_{\delta}} \mu_{\check{c}_{i j}}, \vartheta_{\check{c}_{\delta}} \vartheta_{\check{c}_{i j}}\right\rangle$, therefore

$$
\begin{aligned}
& \operatorname{IFHSWA}\left(\mathfrak{J}_{\check{c}_{11}} \oplus \mathfrak{J}_{\check{c}_{\delta}}, \mathfrak{J}_{\check{c}_{12}} \oplus \mathfrak{J}_{\check{c}_{\delta}}, \ldots, \mathfrak{J}_{\check{c}_{n m}} \oplus \mathfrak{J}_{\check{c}_{\delta}}\right)=\oplus_{j=1}^{m} \gamma_{j}\left(\oplus_{i=1}^{n} \Omega_{i}\left(\mathfrak{J}_{\check{c}_{i j}} \oplus \mathfrak{J}_{\check{c}_{\delta}}\right)\right)_{\Omega_{j}} \\
& =\left\langle 1-\prod_{j=1}^{m}\left(\prod_{i=1}^{n}\left(1-\mu_{\check{c}_{i j}}\right)^{\Omega_{i}}\left(1-\mu_{\check{c}_{\delta}}\right)^{\Omega_{i}}\right)^{\gamma_{j}}, \prod_{j=1}^{m}\left(\prod_{i=1}^{n}\left(\vartheta_{\check{c}_{i j}}\right)^{\Omega_{i}}\left(\vartheta_{\check{c}_{\delta}}\right)^{\Omega_{i}}\right)^{\gamma_{j}}\right\rangle \\
& =\left\langle 1-\left(1-\mu_{\check{c}_{\delta}}\right) \prod_{j=1}^{m}\left(\prod_{i=1}^{n}\left(1-\mu_{\check{c}_{i j}}\right)^{\Omega_{i}}\right)^{\gamma_{j}}, \vartheta_{\check{c}_{\delta}} \prod_{j=1}^{m}\left(\prod_{i=1}^{n}\left(\vartheta_{\check{c}_{i j}}\right)^{\Omega_{i}}\right)^{\gamma_{j}}\right\rangle \\
& =\left\langle 1-\prod_{j=1}^{m}\left(\prod_{i=1}^{n}\left(1-\mu_{\check{c}_{i j}}\right)^{\Omega_{i}}\right)^{\gamma_{j}}, \prod_{j=1}^{m}\left(\prod_{i=1}^{n}\left(\vartheta_{\check{c}_{i j}}\right)^{\Omega_{i}}\right)^{\gamma_{j}}\right\rangle \oplus\left\langle\mu_{\check{c}_{\delta}}, \vartheta_{\check{c}_{\delta}}\right\rangle \\
& =\operatorname{IFHSWA}\left(\mathfrak{J}_{c_{11}}, \mathfrak{J}_{\check{c}_{12}}, \ldots, \mathfrak{J}_{\check{c}_{n m}}\right) \oplus \mathfrak{J}_{\check{c}_{\delta}} .
\end{aligned}
$$

Which completes the proof

\subsection{4. (Homogeneity)}

Prove that IFHSWA $\left(\alpha \mathfrak{J}_{\check{c}_{11}}, \alpha \mathfrak{J}_{\check{c}_{12}}, \ldots, \alpha \mathfrak{J}_{\check{c}_{n m}}\right)=\alpha \operatorname{IFHSWA}\left(\mathfrak{J}_{\check{c}_{11}}, \mathfrak{J}_{\check{c}_{12}}, \ldots, \mathfrak{J}_{\check{c}_{n m}}\right)$ for any positive real number $\alpha$.

Proof. Let $\mathfrak{J}_{\check{c}_{i j}}$ be an IFHSN and $>0$, then by using Definition 5 (3), we have $\alpha \mathfrak{J}_{\check{c}_{i j}}=\left\langle 1-\left(1-\mu_{\check{c}_{i j}}\right)^{\alpha}, \vartheta_{\check{c}_{i j}}{ }^{\alpha}\right\rangle$.

So,

$$
\begin{aligned}
& \operatorname{IFHSWA}\left(\alpha \mathfrak{J}_{\check{c}_{11}}, \alpha \mathfrak{J}_{\check{c}_{12}}, \ldots, \alpha \mathfrak{J}_{\check{c}_{n m}}\right)= \\
& \left\langle 1-\prod_{j=1}^{m}\left(\prod_{i=1}^{n}\left(1-\mu_{\check{c}_{i j}}\right)^{\alpha \Omega_{i}}\right)^{\gamma_{j}}, \prod_{j=1}^{m}\left(\prod_{i=1}^{n}\left(\vartheta_{\check{c}_{i j}}\right)^{\alpha \Omega_{i}}\right)^{\gamma_{j}}\right\rangle \\
& =\left\langle 1-\left(\prod_{j=1}^{m}\left(\prod_{i=1}^{n}\left(1-\mu_{\check{c}_{i j}}\right)^{\Omega_{i}}\right)^{\gamma_{j}}\right)^{\alpha},\left(\prod_{j=1}^{m}\left(\prod_{i=1}^{n}\left(\vartheta_{\check{c}_{i j}}\right)^{\Omega_{i}}\right)^{\gamma_{j}}\right)^{\alpha}\right\rangle \\
& =\alpha \operatorname{IFHSWA}\left(\mathfrak{J}_{\check{c}_{11}}, \mathfrak{J}_{\check{c}_{12}}, \ldots, \mathfrak{J}_{\check{c}_{n m}}\right) .
\end{aligned}
$$

Completes the proof.

Definition 7. Let $\mathfrak{J}_{\check{c}_{k}}=\left(\mu_{\check{c}_{k}}, \vartheta_{\check{c}_{k}}\right)$ be an IFHSN, $\Omega_{i}$ and $\gamma_{j}$ be weight vectors for expert's and multi sub-attributes of the considered attributes correspondingly along with specified circumstances $\Omega_{i}>$ $0, \sum_{i=1}^{n} \Omega_{i}=1, \gamma_{j}>0, \sum_{j=1}^{m} \gamma_{j}=1$. Then IFHSWG operator can be defined as follows:

IFHSWG: $\Delta^{n} \rightarrow \Delta$ defined as follows:

$$
\operatorname{IFHSWG}\left(\mathfrak{J}_{\check{c}_{11}}, \mathfrak{J}_{\check{c}_{12}}, \ldots, \mathfrak{J}_{\check{c}_{n m}}\right)=\otimes_{j=1}^{m}\left(\otimes_{i=1}^{n} \mathfrak{J}_{\check{c}_{n m}}^{\Omega_{i}}\right)^{\gamma_{j}} .
$$


Theorem 2. Let $\mathfrak{J}_{\check{c}_{k}}=\left(\mu_{\check{c}_{k}}, \vartheta_{\check{c}_{k}}\right)$ be an IFHSN. Then, utilizing Equation (7), we get PFHSN and

$$
\begin{gathered}
\text { IFHSWG }\left(\mathfrak{J}_{\check{c}_{11}}, \mathfrak{J}_{\check{c}_{12}}, \ldots, \mathfrak{J}_{\check{c}_{n m}}\right)= \\
\left\langle\prod_{j=1}^{m}\left(\prod_{i=1}^{n}\left(\mu_{\check{c}_{i j}}\right)^{\Omega_{i}}\right)^{\gamma_{j}}, 1-\prod_{j=1}^{m}\left(\prod_{i=1}^{n}\left(1-\vartheta_{\check{c}_{i j}}\right)^{\Omega_{i}}\right)^{\gamma_{j}}\right\rangle
\end{gathered}
$$

$\Omega_{\mathrm{i}}$ and $\gamma_{\mathrm{j}}$ be weight vectors for experts and multi sub-attributes of the considered attributes correspondingly along with specified circumstances $\Omega_{\mathrm{i}}>0, \sum_{\mathrm{i}=1}^{\mathrm{n}} \Omega_{\mathrm{i}}=1, \gamma_{\mathrm{j}}>0, \sum_{\mathrm{j}=1}^{\mathrm{m}} \gamma_{\mathrm{j}}=1$.

Proof. The IFHSWA can be proven as follows utilizing the principle of mathematical induction.

For $n=1$, we get $\Omega_{1}=1$. Then, we have

$$
\begin{aligned}
& \operatorname{IFHSWG}\left(\mathfrak{J}_{\check{c}_{11}}, \mathfrak{J}_{\check{c}_{12}}, \ldots, \mathfrak{J}_{\check{c}_{n m}}\right)=\otimes_{j=1}^{m} \mathfrak{J}_{\check{\mathfrak{c}}_{1 j}}^{\gamma_{j}} \\
& \operatorname{IFHSWG}\left(\mathfrak{J}_{\check{c}_{11}}, \mathfrak{J}_{\check{c}_{12}}, \ldots, \mathfrak{J}_{\check{c}_{n m}}\right)=\left\langle\prod_{j=1}^{m}\left(\mu_{\check{c}_{1 j}}\right)^{\gamma_{j}}, 1-\prod_{j=1}^{m}\left(1-\vartheta_{\check{c}_{1 j}}\right)^{\gamma_{j}}\right\rangle \\
& =\left\langle\prod_{j=1}^{m}\left(\prod_{i=1}^{1}\left(\mu_{\check{c}_{i j}}\right)^{\Omega_{i}}\right)^{\gamma_{j}}, 1-\prod_{j=1}^{m}\left(\prod_{i=1}^{1}\left(1-\vartheta_{\check{c}_{1 j}}\right)^{\Omega_{i}}\right)^{\gamma_{j}}\right\rangle .
\end{aligned}
$$

For $m=1$, we get $\gamma_{1}=1$. Then, we have

$$
\begin{aligned}
& \text { IFHSWG }\left(\mathfrak{J}_{\check{c}_{11}}, \mathfrak{J}_{\check{c}_{12}}, \ldots, \mathfrak{J}_{\check{c}_{n m}}\right)=\otimes_{i=1}^{n}\left(\mathfrak{J}_{\check{c}_{i 1}}\right)^{\Omega_{i}} \\
& =\left\langle\prod_{i=1}^{n}\left(\mu_{\check{c}_{i 1}}\right)^{\Omega_{i}}, 1-\prod_{i=1}^{n}\left(1-\vartheta_{\check{c}_{i 1}}\right)^{\Omega_{i}}\right\rangle \\
& =\left\langle\prod_{j=1}^{1}\left(\prod_{i=1}^{n}\left(\mu_{\check{c}_{i j}}\right)^{\Omega_{i}}\right)^{\gamma_{j}}, 1-\prod_{j=1}^{1}\left(\prod_{i=1}^{n}\left(1-\vartheta_{\check{c}_{i j}}\right)^{\Omega_{i}}\right)^{\gamma_{j}}\right\rangle .
\end{aligned}
$$

This shows that Equation (8) fulfills for $n=1$ and $m=1$. Consider Equation (8) holds for $m=\beta_{1}+1, n=\beta_{2}$ and $m=\beta_{1}, n=\beta_{2}+1$, such as:

$$
\begin{aligned}
& \otimes_{j=1}^{\beta_{1}+1}\left(\otimes_{i=1}^{\beta_{2}}\left(\mathfrak{J}_{\check{c}_{i j}}\right)^{\Omega_{i}}\right)^{\gamma_{j}}=\left\langle\prod_{j=1}^{\beta_{1}+1}\left(\prod_{i=1}^{\beta_{2}}\left(\mu_{\check{c}_{i j}}\right)^{\Omega_{i}}\right)^{\gamma_{j}}, 1-\Pi_{j=1}^{\beta_{1}+1}\left(\Pi_{i=1}^{\beta_{2}}\left(1-\vartheta_{\check{c}_{i j}}\right)^{\Omega_{i}}\right)^{\gamma_{j}}\right\rangle \\
& \otimes_{j=1}^{\beta_{1}}\left(\otimes_{i=1}^{\beta_{2}}\left(\mathfrak{J}_{\check{c}_{i j}}\right)^{\Omega_{i}}\right)^{\gamma_{j}}=\left\langle\prod_{j=1}^{\beta_{1}}\left(\Pi_{i=1}^{\beta_{2}+1}\left(\mu_{\check{c}_{i j}}\right)^{\Omega_{i}}\right)^{\gamma_{j}}, 1-\Pi_{j=1}^{\beta_{1}}\left(\prod_{i=1}^{\beta_{2}+1}\left(1-\vartheta_{\check{c}_{i j}}\right)^{\Omega_{i}}\right)^{\gamma_{j}}\right\rangle
\end{aligned}
$$

For $m=\beta_{1}+1$ and $n=\beta_{2}+1$, we have

$$
\begin{aligned}
& \otimes_{j=1}^{\beta_{1}+1}\left(\otimes_{i=1}^{\beta_{2}+1}\left(\mathfrak{J}_{\check{c}_{i j}}\right)^{\Omega_{i}}\right)^{\gamma_{j}}=\otimes_{j=1}^{\beta_{1}+1}\left(\otimes_{i=1}^{\beta_{2}}\left(\mathfrak{J}_{\check{c}_{i j}}\right)^{\Omega_{i}} \otimes\left(\mathfrak{J}_{\check{c}_{\left(\beta_{2}+1\right) j}}\right)^{\Omega_{\beta_{2}+1}}\right)^{\gamma_{j}} \\
& =\otimes_{j=1}^{\beta_{1}+1}\left(\otimes_{i=1}^{\beta_{2}}\left(\mathfrak{J}_{\check{c}_{i j}}\right)^{\Omega_{i}}\right)^{\gamma_{j}} \otimes_{j=1}^{\beta_{1}+1}\left(\left(\mathfrak{J}_{\check{c}_{\left(\beta_{2}+1\right) j}}\right)^{\Omega_{\beta_{2}+1}}\right)^{\gamma_{j}} \\
& =\left\langle\begin{array}{c}
\prod_{j=1}^{\beta_{1}+1}\left(\prod_{i=1}^{\beta_{2}}\left(\mu_{\check{c}_{i j}}\right)^{\Omega_{i}}\right)^{\gamma_{j}} \otimes \prod_{j=1}^{\beta_{1}+1}\left(\left(\mu_{\check{c}_{\left(\beta_{2}+1\right) j}}\right)^{\Omega_{\beta_{2}+1}}\right)^{\gamma_{j}}, \\
\left.1-\prod_{j=1}^{\beta_{1}+1}\left(\prod_{i=1}^{\beta_{2}}\left(1-\vartheta_{\check{c}_{i j}}\right)^{\Omega_{i}}\right)^{\gamma_{j}} \otimes 1-\prod_{j=1}^{\beta_{1}+1}\left(\left(1-\vartheta_{\check{c}_{\left(\beta_{2}+1\right) j}}\right)^{\Omega_{\beta_{2}+1}}\right)^{\gamma_{j}}\right\rangle
\end{array}\right. \\
& =\prod_{j=1}^{\beta_{1}+1}\left(\prod_{i=1}^{\beta_{2}+1}\left(\mu_{\check{c}_{i j}}\right)^{\Omega_{i}}\right)^{\gamma_{j}}, 1-\prod_{j=1}^{\beta_{1}+1}\left(\prod_{i=1}^{\beta_{2}+1}\left(1-\vartheta_{\check{c}_{i j}}\right)^{\Omega_{i}}\right)^{\gamma_{j}} \text {. }
\end{aligned}
$$

Hence, it is true for $m=\beta_{1}+1$ and $n=\beta_{2}+1$. 
We establish some properties for the collection of IFHSNs based on Theorem 2, by utilizing the proposed IFHSWG operator.

\subsection{Properties of IFHSWG Operator}

3.3.1. (Idempotency)

$\mathfrak{J}_{\check{c}_{i j}}=\mathfrak{J}_{\check{c}_{\delta}}=\left(\mu_{\check{c}_{i j}}, \vartheta_{\check{c}_{i j}}\right) \forall i, j$, then,

$\operatorname{IFHSWG}\left(\mathfrak{J}_{\check{c}_{11}}, \mathfrak{J}_{\check{c}_{12}}, \ldots, \mathfrak{J}_{\check{c}_{n m}}\right)=\mathfrak{J}_{\check{c}_{\delta}}$.

Proof. As we know $\mathfrak{J}_{\check{c}_{i j}}=\mathfrak{J}_{\check{c}_{\delta}}=\left(\mu_{\check{c}_{i j}}, \vartheta_{\check{c}_{i j}}\right)$ to be a collection of IFHSNs, then by Equation (8)

$$
\begin{aligned}
& \operatorname{IFHSWG}\left(\mathfrak{J}_{\check{c}_{11}}, \mathfrak{J}_{\check{c}_{12}}, \ldots, \mathfrak{J}_{\check{c}_{n m}}\right)=\left\langle\prod_{j=1}^{m}\left(\prod_{i=1}^{n}\left(\mu_{\check{c}_{i j}}\right)^{\Omega_{i}}\right)^{\gamma_{j}}, 1-\prod_{j=1}^{m}\left(\prod_{i=1}^{n}\left(1-\vartheta_{\check{c}_{i j}}\right)^{\Omega_{i}}\right)^{\gamma_{j}}\right\rangle \\
& =\left\langle\left(\left(\mu_{\check{c}_{i j}}\right)^{\sum_{i=1}^{n} \Omega_{i}}\right)^{\sum_{j=1}^{m} \gamma_{j}}, 1-\left(\left(1-\vartheta_{\check{c}_{i j}}\right)^{\sum_{i=1}^{n} \Omega_{i}}\right)^{\sum_{j=1}^{m} \gamma_{j}}\right\rangle \\
& =\left\langle\mu_{\check{c}_{i j}}, 1-\left(1-\vartheta_{\check{c}_{i j}}\right)\right\rangle=\left(\mu_{\check{c}_{i j}}, \vartheta_{\check{c}_{i j}}\right)=\mathfrak{J}_{\check{c}_{\delta}} .
\end{aligned}
$$

\subsection{2. (Boundedness)}

Let $\mathfrak{J}_{\check{c}_{i j}}$ be a collection of IFHSNs and $\left.\mathfrak{J}_{\check{c}_{i j}}-=\left\langle\begin{array}{cc}\min & \min \\ j & i\end{array} \mu_{\check{c}_{i j}}\right\}, \quad \underset{j}{\max } \max \left\{\vartheta_{\check{c}_{i j}}\right\}\right\rangle$ and $\mathfrak{J}_{\check{c}_{i j}}+$

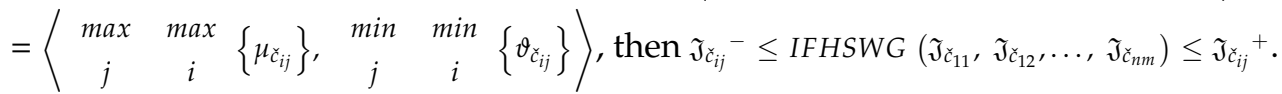

Proof. As we know that $\mathfrak{J}_{\check{c}_{i j}}=\left(\mu_{\check{c}_{i j}}, \mathcal{J}_{\check{c}_{i j}}\right)$ is an IFHSN, then

$$
\begin{aligned}
& \min _{j} \min _{i}\left\{\mu_{\check{c}_{i j}}\right\} \leq \mu_{\check{c}_{i j}} \leq \max _{j}^{\max }\left\{\mu_{\check{c}_{i j}}\right\} \\
& \Rightarrow \min _{j} \min _{i}\left\{\mu_{\check{c}_{i j}}\right\} \leq \mu_{\check{c}_{i j}} \leq \max _{j} \max _{i}\left\{\mu_{\check{c}_{i j}}\right\} \\
& \Leftrightarrow\left(\begin{array}{cc}
\min & \min \\
j & i
\end{array}\left\{\mu_{\check{c}_{i j}}\right\}\right)^{\Omega_{i}} \leq\left(\mu_{\check{c}_{i j}}\right)^{\Omega_{i}} \leq\left(\begin{array}{cc}
\max & \max \\
j & i
\end{array}\left\{\mu_{\check{c}_{i j}}\right\}\right)^{\Omega_{i}}
\end{aligned}
$$

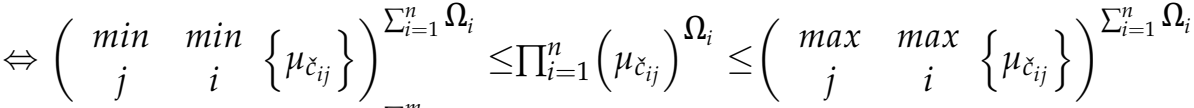

$$
\begin{aligned}
& \Leftrightarrow\left(\begin{array}{cc}
\min & \min \\
j & i
\end{array}\left\{\mu_{\check{c}_{i j}}\right\}\right)^{\sum_{j=1}^{m} \gamma_{j}} \leq \prod_{j=1}^{m}\left(\prod_{i=1}^{n}\left(\mu_{\check{c}_{i j}}\right)^{\Omega_{i}}\right)^{\gamma_{j}} \leq\left(\begin{array}{cc}
\max & \max \\
j & i
\end{array}\left\{\mu_{\check{c}_{i j}}\right\}\right)^{\sum_{j=1}^{m} \gamma_{j}} \\
& \Leftrightarrow \min _{j} \min _{i}\left\{\mu_{\check{c}_{i j}}\right\} \leq \prod_{j=1}^{m}\left(\prod_{i=1}^{n}\left(\mu_{\check{c}_{i j}}\right)^{\Omega_{i}}\right)^{\gamma_{j}} \leq \underset{j}{\max } \underset{i}{\max }\left\{\mu_{\check{c}_{i j}}\right\} \\
& \Leftrightarrow \min _{j} \min _{i}\left\{\mu_{\check{c}_{i j}}\right\} \leq \prod_{j=1}^{m}\left(\prod_{i=1}^{n}\left(\mu_{\check{c}_{i j}}\right)^{\Omega_{i}}\right)^{\gamma_{j}} \leq \underset{j}{\max } \max \left\{\mu_{\check{c}_{i j}}\right\} \\
& \Leftrightarrow \min _{j} \min _{i}\left\{\mu_{\check{c}_{i j}}\right\} \leq \prod_{j=1}^{m}\left(\prod_{i=1}^{n}\left(\mu_{\check{c}_{i j}}\right)^{\Omega_{i}}\right)^{\gamma_{j}} \leq \underset{j}{\max } \underset{i}{\max }\left\{\mu_{\check{c}_{i j}}\right\}
\end{aligned}
$$

Similarly,

$$
\Leftrightarrow \min _{j} \min _{i}\left\{\vartheta_{\check{c}_{i j}}\right\} \leq 1-\prod_{j=1}^{m}\left(\prod_{i=1}^{n}\left(1-\vartheta_{\check{c}_{i j}}\right)^{\Omega_{i}}\right)^{\gamma_{j}} \leq \max _{j}^{\max }\left\{\vartheta_{\check{c}_{i j}}\right\}
$$


Let IFHSWG $\left(\mathfrak{J}_{\check{c}_{11}}, \mathfrak{J}_{\check{c}_{12}}, \ldots, \mathfrak{J}_{\check{c}_{n m}}\right)=\left\langle\mu_{\check{c}_{\delta}}, \vartheta_{\check{c}_{\delta}}\right\rangle=\mathfrak{J}_{\check{c}_{\delta}}$, then Inequalities 9 and 10 can be transformed into the following form: $\underset{j}{\min } \min \left\{\mu_{\check{c}_{i j}}\right\} \leq \mu_{\check{c}_{\delta}} \leq \underset{j}{\max } \max \left\{\mu_{\check{c}_{i j}}\right\}$ and $\min _{j} \min _{i}\left\{\vartheta_{\boldsymbol{x}_{i j}}\right\} \leq \vartheta_{\boldsymbol{\tau}_{\delta}} \leq \underset{j}{\max } \max _{i}\left\{\vartheta_{\boldsymbol{\tau}_{i j}}\right\}$ respectively.

So, by utilizing Equation (1), we have:

$\mathbb{S}\left(\mathfrak{J}_{\check{c}_{\delta}}\right)=\mu_{\check{c}_{\delta}}-\vartheta_{\check{c}_{\delta}} \leq \max _{j} \max _{i}\left\{\mu_{\check{c}_{i j}}\right\}-\min _{j} \min _{i}\left\{\vartheta_{\check{c}_{i j}}\right\}=\mathbb{S}\left(\mathfrak{J}_{\breve{c}_{i j}}{ }^{+}\right)$,

$\mathbb{S}\left(\mathfrak{J}_{\check{c}_{\delta}}\right)=\mu_{\check{c}_{\delta}}-\vartheta_{\check{c}_{\delta}} \geq \min _{j} \min _{i}\left\{\mu_{\check{c}_{\delta}}\right\}-\underset{j}{\max } \max \left\{\vartheta_{\check{c}_{\delta}}\right\}=\mathbb{S}\left(\mathfrak{J}_{\check{c}_{i j}}{ }^{-}\right)$. Then, by order relation among two IFHSNs, we have

$\mathfrak{J}_{\check{c}_{i j}}{ }^{-} \leq \operatorname{IFHSWG}\left(\mathfrak{J}_{\check{c}_{11}}, \mathfrak{J}_{\check{c}_{12}}, \ldots, \mathfrak{J}_{\check{c}_{n m}}\right) \leq \mathfrak{J}_{\check{c}_{i j}}{ }^{+}$.

\subsection{3. (Shift Invariance)}

If $\mathfrak{J}_{\check{c}_{\delta}}=\left\langle\mu_{\check{c}_{\delta}}, \vartheta_{\check{c}_{\delta}}\right\rangle$ be an IFHSN. Then,

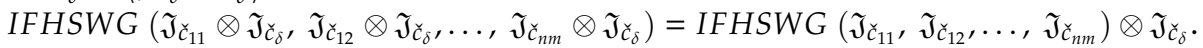

Proof. Let $\mathfrak{J}_{\check{c}_{\delta}}$ and $\mathfrak{J}_{\check{c}_{i j}}$ be two IFHSNs. Utilizing Definition 5 (2), we have: $\mathfrak{J}_{\check{c}_{\delta}} \otimes \mathfrak{J}_{c_{i j}}=\left\langle\mu_{\check{c}_{11}} \mu_{\check{c}_{12}}, \vartheta_{\check{c}_{11}}+\vartheta_{\check{c}_{12}}-\vartheta_{\check{c}_{11}} \vartheta_{\check{c}_{12}}\right\rangle$. Therefore

$$
\begin{aligned}
& \operatorname{IFHSWG}\left(\mathfrak{J}_{\check{c}_{11}} \otimes \mathfrak{J}_{\check{c}_{\delta}}, \mathfrak{J}_{\check{c}_{12}} \otimes \mathfrak{J}_{\check{c}_{\delta}}, \ldots, \mathfrak{J}_{\check{c}_{n m}} \otimes \mathfrak{J}_{\check{c}_{\delta}}\right)=\otimes_{j=1}^{m} \gamma_{j}\left(\otimes_{i=1}^{n} \Omega_{i}\left(\mathfrak{J}_{\check{c}_{i j}} \otimes \mathfrak{J}_{\check{c}_{\delta}}\right)\right)_{\check{c}} \\
& =\left\langle\prod_{j=1}^{m}\left(\prod_{i=1}^{n}\left(\mu_{\check{c}_{i j}}\right)^{\Omega_{i}}\left(\mu_{\check{c}_{\delta}}\right)^{\Omega_{i}}\right)^{\gamma_{j}}, 1-\prod_{j=1}^{m}\left(\prod_{i=1}^{n}\left(1-\vartheta_{\check{c}_{i j}}\right)^{\Omega_{i}}\left(1-\vartheta_{\check{c}_{\delta}}\right)^{\Omega_{i}}\right)^{\gamma_{j}}\right\rangle \\
& =\left\langle\mu_{\check{c}_{\delta}} \prod_{j=1}^{m}\left(\prod_{i=1}^{n}\left(\mu_{\check{c}_{i j}}\right)^{\Omega_{i}}\right)^{\gamma_{j}}, 1-\left(1-\vartheta_{\check{c}_{\delta}}\right) \prod_{j=1}^{m}\left(\prod_{i=1}^{n}\left(1-\vartheta_{\check{c}_{i j}}\right)^{\Omega_{i}}\right)^{\gamma_{j}}\right\rangle \\
& =\left\langle\prod_{j=1}^{m}\left(\prod_{i=1}^{n}\left(\mu_{\check{c}_{i j}}\right)^{\Omega_{i}}\right)^{\gamma_{j}}, 1-\prod_{j=1}^{m}\left(\prod_{i=1}^{n}\left(1-\vartheta_{\check{c}_{i j}}\right)^{\Omega_{i}}\right)^{\gamma_{j}}\right\rangle \otimes\left\langle\mu_{\check{c}_{\delta}}, \vartheta_{\check{c}_{\delta}}\right\rangle \\
& =\operatorname{IFHSWG}\left(\mathfrak{J}_{c_{11}}, \mathfrak{J}_{\check{c}_{12}}, \ldots, \mathfrak{J}_{\check{c}_{n m}}\right) \otimes \mathfrak{J}_{\check{c}_{\delta}} .
\end{aligned}
$$

Which completes the proof.

\subsection{4. (Homogeneity)}

Prove that IFHSWG $\left(\alpha \mathfrak{J}_{\check{c}_{11}}, \alpha \mathfrak{J}_{\check{c}_{12}}, \ldots, \alpha \mathfrak{J}_{\check{c}_{n m}}\right)=\alpha \operatorname{IFHSWG}\left(\mathfrak{J}_{\check{c}_{11}}, \mathfrak{J}_{\check{c}_{12}}, \ldots, \mathfrak{J}_{\check{c}_{n m}}\right)$ for any positive real number $\alpha$.

Proof. Similar to Section 3.2.4.

\section{Multi-Criteria Decision-Making Approach under IFHSS Information}

An MCDM technique is constructed here with underdeveloped operators and described as a numerical illustration to demonstrating their competence. 


\subsection{Proposed Approach to Solve the MCDM Problem}

Consider $\mathcal{Q}=\left\{\mathcal{Q}^{1}, \mathcal{Q}^{2}, \mathcal{Q}^{3}, \ldots, \mathcal{Q}^{s}\right\}$ to be a set of $s$ alternatives and $\mathcal{X}=\left\{\mathcal{X}_{1}, \mathcal{X}_{2}, \mathcal{X}_{3}, \ldots, \mathcal{X}_{n}\right\}$ to be a set of $n$ experts. The weights of experts are given as $\Omega=\left(\Omega_{1}, \Omega_{1}, \ldots, \Omega_{n}\right)^{T}$ and $\Omega_{i}>0, \sum_{i=1}^{n} \Omega_{i}=1$. Let $\mathfrak{L}=\left\{c_{1}, c_{2}, \ldots, c_{m}\right\}$ be a set of attributes with their corresponding multi sub-attributes such as $\mathfrak{L}^{\prime}=\left\{\left(c_{1 \rho} \times c_{2 \rho} \times \ldots \times c_{m \rho}\right)\right.$ for all $\left.\rho \in\{1,2, \ldots, t\}\right\}$ with weights $\gamma=\left(\gamma_{1 \rho}, \gamma_{2 \rho}, \gamma_{3 \rho}, \ldots, \gamma_{m \rho}\right)^{T}$ such as $\gamma_{\rho}>0, \sum_{\rho=1}^{t} \gamma_{\rho}=1$. The components in the collection of sub-attributes are multi-valued; for the sake of accessibility, the components of $\mathfrak{L}^{\prime}$ can be stated as $\mathfrak{L}^{\prime}=\left\{\check{c}_{\partial}: \partial \in\{1,2, \ldots, k\}\right\}$. The team of experts $\left\{\mathcal{X}_{i}: i=1,2, \ldots\right.$, $n\}$ appraise the alternatives $\left\{\mathcal{Q}^{(z)}: z=1,2, \ldots, s\right\}$ under the preferred sub-attributes of the considered parameters $\left\{\check{c}_{\partial}: \partial=1,2, \ldots, k\right\}$ given in the form of IFHSNs such as $\left(\mathfrak{J}_{\tilde{c}_{i j}}^{(z)}\right)_{n \times \partial}=\left(\mu_{\check{c}_{i j}}^{(z)}, \vartheta_{\check{c}_{i j}}^{(z)}\right)_{n \times \partial}$, where $0 \leq \mu_{\tilde{c}_{i j}}^{(z)}, \vartheta_{\tilde{c}_{i j}}^{(z)} \leq 1$ and $\mu_{\tilde{c}_{i j}}^{(z)}+\vartheta_{\tilde{c}_{i j}}^{(z)} \leq 1$ for all $i, j$.

\subsection{Algorithm for Developed Aggregation Operators under IFHSS Information}

Step 1. Acquire a decision matrix from experts for each alternative $\left\{\mathcal{Q}^{(z)}: z=1,2, \ldots, s\right\}$ such as follows:

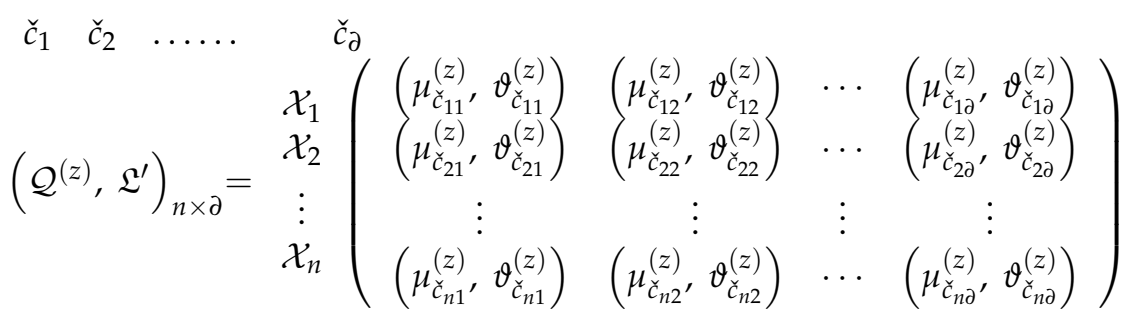

Step 2. Transforming cost type sub-attributes to benefit type sub-attribute using the normalization rule and obtain the normalized decision matrix.

$$
\left\langle i j=\left\{\begin{array}{l}
\mathfrak{J}_{\mathfrak{c}_{i j}}^{c}=\left(\vartheta_{\tilde{c}_{i j}}^{(z)}, \mu_{\check{c}_{i j}}^{(z)}\right) ; \quad \text { cost type parameter } \\
\mathfrak{J}_{\check{c}_{i j}}=\left(\mu_{\tilde{c}_{i j}}^{(z)}, \vartheta_{\check{c}_{i j}}^{(z)}\right) ; \text { benefit type parameter }
\end{array}\right.\right.
$$

Step 3. Utilizing the developed aggregate, operators obtained a collective decision matrix $\mathfrak{J}_{\check{c}_{i j}}$ for each alternative $\mathcal{T}=\left\{\mathcal{T}^{1}, \mathcal{T}^{2}, \mathcal{T}^{3}, \ldots, \mathcal{T}^{s}\right\}$.

Step 4. If $\mathcal{T}=\left\{\mathcal{T}^{1}, \mathcal{T}^{2}, \mathcal{T}^{3}, \ldots, \mathcal{T}^{s}\right\}$ are a collection of considered alternatives, then calculate the score values utilizing Equation (1).

Step 5. Pick the most suitable alternate with a supreme score value.

Step 6. Rank the alternatives. 
The above presented approach can be expressed graphically in Figure 1 such as follows:

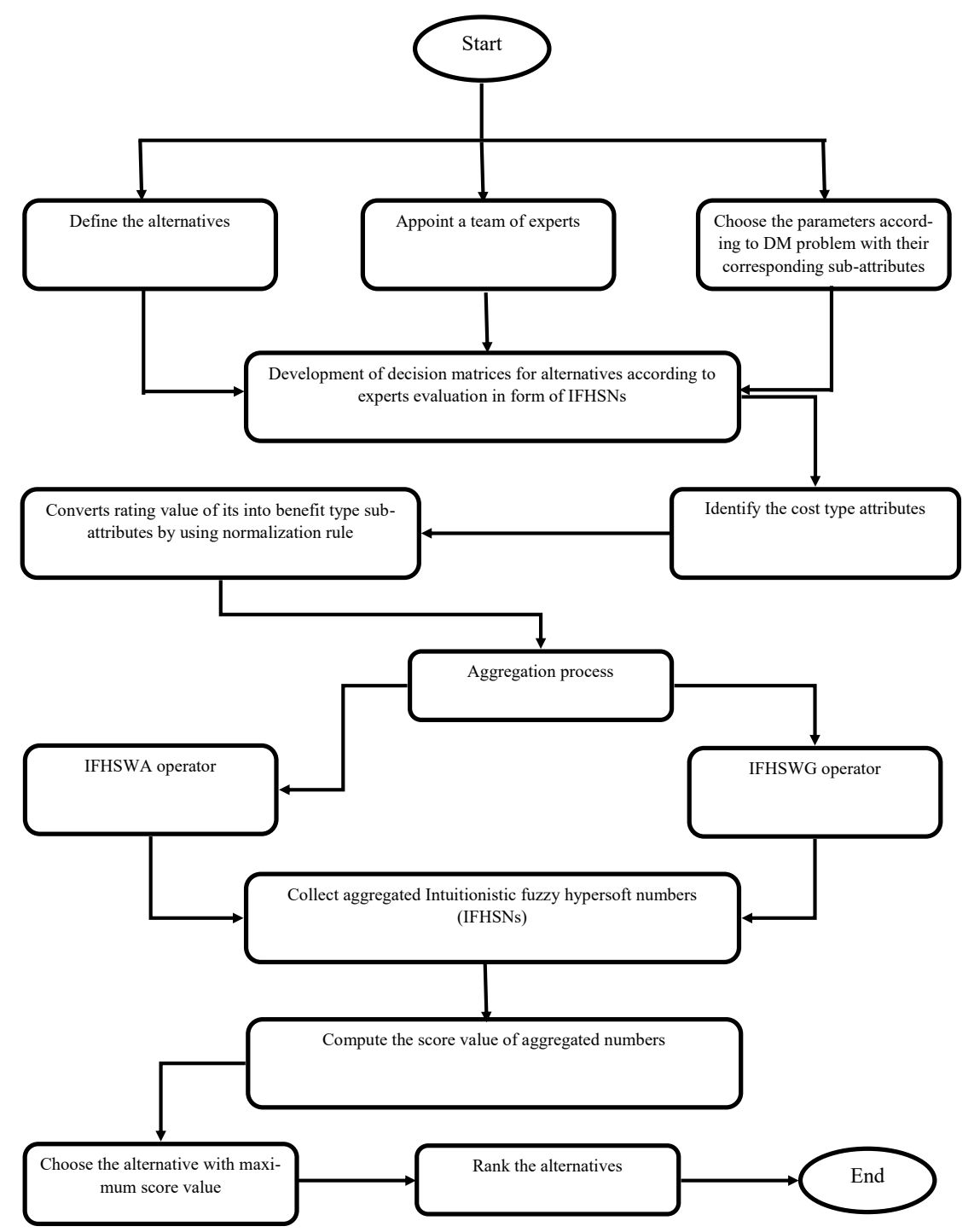

Figure 1. Flow chart of the presented decision-making approach.

\subsection{Case Study}

The problem of supplier selection is important in both methodical and practical dimensions. It is a key issue for the firm as the optimal choice of supplier is the basis for effective management of the supply chain and the source of competitive advantage. Including pro-environmental imperatives and other aspects of sustainable development in the supplier selection process makes the correct supplier selection difficult and multidimensional. Depending on the scope of pro-environmental or pro-social activities, supplier selection is often referred to in the literature as "sustainable supplier selection". It is a complex and multidimensional problem, where there are conflicting criteria, and the evaluation process itself requires consideration of many perspectives. From these points of view, the supplier selection problem is often treated in the literature as a "reference" problem, where multi-criteria decision support methods (MCDM) have been widely applied. The problem of selecting and evaluating a sustainable supplier is addressed in many works. Analyzing papers [49-51] and using review papers [52-54] in this example of sustainable supplier selection, it was decided to extract the following set of five basic criteria. These are: $c_{1}$, quality of service; $c_{2}$, pollution control; $c_{3}$, environmental efficiency; $c_{4}$, price; and $c_{5}$, corporate social responsibility. 
Let $\left\{\mathcal{X}^{(1)}, \mathcal{X}^{(2)}, \mathcal{X}^{(3)}, \mathcal{X}^{(4)}, \mathcal{X}^{(5)}\right\}$ be a set of substitutes and $\mathfrak{L}=$ $\left\{c_{1}=\right.$ Superiority, $c_{2}=$ Delivery, $c_{3}=$ Services, $c_{4}=$ Troposphere, $c_{5}=$ Commercial societal concern $\}$ be a collection of considered attributes given as Superiority $=c_{1}=\left\{c_{11}=\right.$ national level, $c_{12}=$ international level $\}$, Delivery $=c_{2}=\left\{c_{21}=\right.$ by carriar, $c_{22}=$ by hand $\}$, Services $=c_{3}=\left\{c_{31}=\right.$ services $\}$, Troposphere $=c_{4}=\left\{c_{41}=\right.$ friendly, $c_{42}=$ non serious $\}$, and Commercial societal concern $=c_{5}=\left\{c_{51}=\right.$ Commercial societal concern $\}$. Let $\mathfrak{L}^{\prime}=c_{1} \times c_{2} \times c_{3} \times c_{4} \times c_{5}$ be a set of sub-attributes

$$
\begin{aligned}
& \mathfrak{L}^{\prime}=c_{1} \times c_{2} \times c_{3} \times c_{4} \times c_{5}=\left\{c_{11}, c_{12}\right\} \times\left\{c_{21}, c_{22}\right\} \times\left\{c_{31}, c_{32}\right\} \times\left\{c_{41}\right\} \times\left\{c_{51}\right\} \\
& =\left\{\begin{array}{l}
\left(c_{11}, c_{21}, c_{31}, c_{41}, c_{51}\right),\left(c_{11}, c_{21}, c_{32}, c_{41}, c_{51}\right),\left(c_{11}, c_{22}, c_{31}, c_{41}, c_{51}\right),\left(c_{11}, c_{22}, c_{32}, c_{41}, c_{51}\right), \\
\left(c_{12}, c_{21}, c_{31}, c_{41}, c_{51}\right),\left(c_{12}, c_{21}, c_{32}, c_{41}, c_{51}\right),\left(c_{12}, c_{22}, c_{31}, c_{41}, c_{51}\right),\left(c_{12}, c_{22}, c_{32}, c_{41}, c_{51}\right)
\end{array}\right\}
\end{aligned}
$$

$\mathfrak{L}^{\prime}=\left\{\check{c}_{1}, \check{c}_{2}, \check{c}_{3}, \check{c}_{4}, \check{c}_{5}, \check{c}_{6}, \check{c}_{7}, \check{c}_{8}\right\}$ be a set of all sub-attributes with weights $(0.12,0.18,0.1,0.15,0.05,0.22,0.08,0.1)^{T}$. Consider $\left\{\mathcal{Q}^{(1)}, \mathcal{Q}^{(2)}, \mathcal{Q}^{(3)}, \mathcal{Q}^{(4)}\right\}$ be a set of experts with weights $(0.2,0.3,0.4,0.1)^{T}$ to assess the finest alternate. Experts give their preferences in terms of IFHSNs using considered multi sub-attributes. The following is that the procedure progressed to get the most productive choice.

\subsection{Sustainable Supplier Selection Using Intuitionistic Fuzzy Hypersoft Weighted Average Operator}

Step 1. Experts examine the circumstances in the instance of IFHSNs. The multi subattributes of the considered attributes, along with a summary of their score values are given in Tables 1-4.

Table 1. Decision Matrix for Alternative $\mathcal{Q}^{(1)}$.

\begin{tabular}{ccccccccc}
\hline $\mathcal{Q}^{(1)}$ & $\check{\boldsymbol{c}}_{1}$ & $\check{\boldsymbol{c}}_{2}$ & $\check{\boldsymbol{c}}_{3}$ & $\check{\boldsymbol{c}}_{4}$ & $\check{\boldsymbol{c}}_{5}$ & $\check{\boldsymbol{c}}_{6}$ & $\check{\boldsymbol{c}}_{7}$ & $\check{\boldsymbol{c}}_{8}$ \\
\hline $\mathcal{X}_{1}$ & $(0.3,0.5)$ & $(0.2,0.3)$ & $(0.1,0.3)$ & $(0.3,0.6)$ & $(0.2,0.4)$ & $(0.2,0.6)$ & $(0.5,0.4)$ & $(0.1,0.3)$ \\
$\mathcal{X}_{2}$ & $(0.2,0.7)$ & $(0.4,0.6)$ & $(0.3,0.4)$ & $(0.1,0.2)$ & $(0.1,0.2)$ & $(0.2,0.4)$ & $(0.2,0.5)$ & $(0.4,0.5)$ \\
$\mathcal{X}_{3}$ & $(0.2,0.3)$ & $(0.2,0.5)$ & $(0.1,0.6)$ & $(0.3,0.4)$ & $(0.4,0.6)$ & $(0.1,0.4)$ & $(0.2,0.3)$ & $(0.2,0.5)$ \\
$\mathcal{X}_{4}$ & $(0.2,0.4)$ & $(0.2,0.3)$ & $(0.2,0.4)$ & $(0.4,0.6)$ & $(0.3,0.5)$ & $(0.3,0.6)$ & $(0.4,0.5)$ & $(0.1,0.3)$ \\
\hline
\end{tabular}

Table 2. Decision Matrix for Alternative $\mathcal{Q}^{(2)}$.

\begin{tabular}{ccccccccc}
\hline $\mathcal{Q}^{(2)}$ & $\check{\boldsymbol{c}}_{1}$ & $\check{\boldsymbol{c}}_{2}$ & $\check{\boldsymbol{c}}_{3}$ & $\check{\boldsymbol{c}}_{4}$ & $\check{\boldsymbol{c}}_{5}$ & $\check{\boldsymbol{c}}_{6}$ & $\check{\boldsymbol{c}}_{7}$ & $\check{\boldsymbol{c}}_{8}$ \\
\hline $\mathcal{X}_{1}$ & $(0.2,0.6)$ & $(0.3,0.4)$ & $(0.4,0.5)$ & $(0.3,0.5)$ & $(0.5,0.4)$ & $(0.4,0.6)$ & $(0.3,0.5)$ & $(0.4,0.5)$ \\
$\mathcal{X}_{2}$ & $(0.3,0.5)$ & $(0.2,0.4)$ & $(0.1,0.2)$ & $(0.1,0.2)$ & $(0.4,0.5)$ & $(0.1,0.3)$ & $(0.2,0.7)$ & $(0.1,0.8)$ \\
$\mathcal{X}_{3}$ & $(0.3,0.7)$ & $(0.4,0.5)$ & $(0.2,0.8)$ & $(0.3,0.4)$ & $(0.2,0.3)$ & $(0.3,0.4)$ & $(0.1,0.2)$ & $(0.7,0.2)$ \\
$\mathcal{X}_{4}$ & $(0.5,0.4)$ & $(0.1,0.6)$ & $(0.2,0.3)$ & $(0.2,0.3)$ & $(0.1,0.2)$ & $(0.2,0.4)$ & $(0.4,0.6)$ & $(0.5,0.5)$ \\
\hline
\end{tabular}

Table 3. Decision Matrix for Alternative $\mathcal{Q}^{(3)}$.

\begin{tabular}{ccccccccc}
\hline $\mathcal{Q}^{(3)}$ & $\check{\boldsymbol{c}}_{1}$ & $\check{\boldsymbol{c}}_{2}$ & $\check{\boldsymbol{c}}_{3}$ & $\check{\boldsymbol{c}}_{4}$ & $\check{\boldsymbol{c}}_{5}$ & $\check{\boldsymbol{c}}_{6}$ & $\check{\boldsymbol{c}}_{7}$ & $\check{\boldsymbol{c}}_{8}$ \\
\hline $\mathcal{X}_{1}$ & $(0.4,0.5)$ & $(0.3,0.5)$ & $(0.4,0.5)$ & $(0.3,0.4)$ & $(0.2,0.4)$ & $(0.4,0.5)$ & $(0.3,0.4)$ & $(0.3,0.5)$ \\
$\mathcal{X}_{2}$ & $(0.3,0.4)$ & $(0.1,0.3)$ & $(0.1,0.8)$ & $(0.1,0.2)$ & $(0.4,0.6)$ & $(0.3,0.7)$ & $(0.6,0.1)$ & $(0.8,0.1)$ \\
$\mathcal{X}_{3}$ & $(0.6,0.2)$ & $(0.3,0.4)$ & $(0.7,0.3)$ & $(0.3,0.4)$ & $(0.1,0.2)$ & $(0.4,0.5)$ & $(0.3,0.5)$ & $(0.6,0.3)$ \\
$\mathcal{X}_{4}$ & $(0.5,0.4)$ & $(0.2,0.3)$ & $(0.4,0.6)$ & $(0.3,0.4)$ & $(0.3,0.6)$ & $(0.7,0.2)$ & $(0.4,0.2)$ & $(0.5,0.2)$ \\
\hline
\end{tabular}

Table 4. Decision Matrix for Alternative $\mathcal{Q}^{(4)}$.

\begin{tabular}{ccccccccc}
\hline $\mathcal{Q}^{(4)}$ & $\check{\boldsymbol{c}}_{1}$ & $\check{\boldsymbol{c}}_{2}$ & $\check{\boldsymbol{c}}_{3}$ & $\check{\boldsymbol{c}}_{4}$ & $\check{\boldsymbol{c}}_{5}$ & $\check{\boldsymbol{c}}_{6}$ & $\check{\boldsymbol{c}}_{7}$ & \multicolumn{1}{c}{$\check{\boldsymbol{c}}_{8}$} \\
\hline $\mathcal{X}_{1}$ & $(0.2,0.7)$ & $(0.4,0.5)$ & $(0.2,0.4)$ & $(0.4,0.3)$ & $(0.1,0.2)$ & $(0.2,0.4)$ & $(0.3,0.4)$ & $(0.2,0.4)$ \\
$\mathcal{X}_{2}$ & $(0.3,0.5)$ & $(0.2,0.4)$ & $(0.8,0.1)$ & $(0.5,0.2)$ & $(0.4,0.3)$ & $(0.4,0.5)$ & $(0.7,0.2)$ & $(0.6,0.3)$ \\
$\mathcal{X}_{3}$ & $(0.6,0.3)$ & $(0.4,0.5)$ & $(0.6,0.2)$ & $(0.6,0.4)$ & $(0.1,0.2)$ & $(0.3,0.4)$ & $(0.5,0.3)$ & $(0.40 .5)$ \\
$\mathcal{X}_{4}$ & $(0.5,0.4)$ & $(0.1,0.3)$ & $(0.3,0.5)$ & $(0.5,0.3)$ & $(0.3,0.5)$ & $(0.8,0.1)$ & $(0.3,0.5)$ & $(0.2,0.5)$ \\
\hline
\end{tabular}


Step 2. There is no need for normalization, as all attributes are identical.

Step 3. Using Equation (4), expert's opinion can be summarized as follows: $\mathcal{L}_{1}=\langle 0.23054,0.42563\rangle, \mathcal{L}_{2}=\langle 0.291197,0.415560\rangle, \mathcal{L}_{3}=\langle 0.447878,0.363226\rangle$, and $\mathcal{L}_{4}=\langle 0.441142,0.345641\rangle$.

Step 4. Calculate the score values using Equation (1). $\mathbb{S}\left(\mathcal{L}_{1}\right)=-0.195086$, $\mathbb{S}\left(\mathcal{L}_{2}\right)=-0.124363, \mathbb{S}\left(\mathcal{L}_{3}\right)=0.084652$, and $\mathbb{S}\left(\mathcal{L}_{4}\right)=0.095501$.

Step 5. By using the obtained score values, we can see that $\mathcal{Q}^{(4)}$ is the best alternative.

Step 6. Hence, the following is an arrangement of alternatives: $\mathbb{S}\left(\mathcal{L}_{4}\right)>\mathbb{S}\left(\mathcal{L}_{3}\right)>\mathbb{S}\left(\mathcal{L}_{2}\right)$ $>\mathbb{S}\left(\mathcal{L}_{1}\right)$. So, $\mathcal{Q}^{(4)}>\mathcal{Q}^{(3)}>\mathcal{Q}^{(2)}>\mathcal{Q}^{(1)}$, hence, the alternative $\mathcal{Q}^{(4)}$ is the most suitable alternative.

\subsection{Sustainable Supplier Selection Using the Intuitionistic Fuzzy Hypersoft Weighted Geometric Operator}

Step 1. Experts examine the circumstances in the instance of IFHSNs. The multi subattributes of the considered attributes, along with a summary of their score values are given in Tables $1-4$.

Step 2. There is no need for normalization, as all attributes are identical.

Step 3. Using Equation (8), expert's opinion can be summarized as follows: $\mathcal{L}_{1}=\langle 0.201387,0.461254\rangle, \mathcal{L}_{2}=\langle 0.238098,0.480574\rangle, \mathcal{L}_{3}=\langle 0.317227,0.459177\rangle$, and $\mathcal{L}_{4}=\langle 0.366312,0.402225\rangle$.

Step 4. Calculate the score values using Equation (1). $\mathbb{S}\left(\mathcal{L}_{1}\right)=-0.259867$, $\mathbb{S}\left(\mathcal{L}_{2}\right)=-0.242376, \mathbb{S}\left(\mathcal{L}_{3}\right)=-0.141950$, and $\mathbb{S}\left(\mathcal{L}_{4}\right)=-0.035913$.

Step 5. By using the obtained score values, we can see that $\mathcal{Q}^{(4)}$ is the best alternative.

Step 6. Hence, the following is an arrangement of alternatives $\mathbb{S}\left(\mathcal{L}_{4}\right)>\mathbb{S}\left(\mathcal{L}_{3}\right)>\mathbb{S}\left(\mathcal{L}_{2}\right)$ $>\mathbb{S}\left(\mathcal{L}_{1}\right)$. So, $\mathcal{Q}^{(4)}>\mathcal{Q}^{(3)}>\mathcal{Q}^{(2)}>\mathcal{Q}^{(1)}$, therefore, the alternate $\mathcal{Q}^{(4)}$ is the most appropriate alternative.

\section{Comparative Analysis and Discussion}

In the subsequent section, we are going to talk over the usefulness, easiness, and manageability of the assistance of the planned method. We also performed an ephemeral evaluation of the undermentioned: the planned technique, along with some prevailing methodologies.

\subsection{Superiority of the Proposed Method}

Through this study and comparison, it could be determined that the consequences acquired by the suggested approach have been more common than either available methods. However, overall, the DM procedure associated with the prevailing DM methods accommodates extra information to address hesitation. In addition, FS's various hybrid structures are becoming a special feature of IFHSS, along with some appropriate circumstances which have been added. The general information associated with the object can be stated precisely and analytically (see Table 5). Therefore, it is a suitable technique to syndicate inaccurate and ambiguous information in the DM process. Hence, the suggested approach is practical, modest, and in advance of fuzzy sets' distinctive hybrid structures.

\subsection{Discussion}

Using MD, Zadeh's [1] FS only handled the inexact and imprecise information of sub-attributes of considered attributes for each alternative. However, the FS has no evidence regarding the NMD of the considered parameters. The existing FS only contracts the ambiguous difficulties using MD, though our planned technique accommodates the vagueness applying MD and NMD. Additionally, Zhang et al. [55] and Xu et al. [56] devel- 
oped IFS which deals with the vague information using MD and NMD. However, these theories are unable to deal with the parametric values of the alternatives. Maji et al. [7] presented the notion of FSS to deal with the parametrization of the objects which contain uncertainty by considering the MD of the attributes. However, the presented FSS provides no information about the NMD of the object. To overcome the presented drawback, Maji et al. [9] offered the concept of IFSS; the presented notion handles the uncertain object more accurately, utilizing the MD and NMD of the attributes with their parametrization and $M D+N M D \leq 1$. All the above mentioned studies have no information about the sub-attributes of the considered attributes. Therefore, the above mentioned theories are unable to handle the scenario when attributes have their corresponding sub-attributes. Our presented approach can resolve these complications simply, and bring additional operational consequences in the DM method. It may be seen that the best selection of the suggested approach is to resemble the verbalized own method, and this ensures the liableness along with the effectiveness of the recommended approach.

Table 5. Comparison of IFHSSs with some prevailing studies.

\begin{tabular}{|c|c|c|c|c|c|c|c|c|}
\hline & Set & Truthiness & Falsity & Attributes & Sub-Attributes & $\begin{array}{c}\text { Loss of } \\
\text { Information }\end{array}$ & Parametrization & Advantages \\
\hline Zadeh [1] & FS & $\checkmark$ & $\times$ & $\checkmark$ & $x$ & $\times$ & $\begin{array}{l}\mathrm{D} \\
\mathrm{us}\end{array}$ & $\begin{array}{l}\text { als uncertainty by } \\
\text { ing fuzzy interval }\end{array}$ \\
\hline Maji et al. [7] & FSS & $\checkmark$ & $x$ & $\checkmark$ & $x$ & $x$ & $\begin{array}{l}\mathrm{D} \\
\text { usin }\end{array}$ & $\begin{array}{l}\text { als uncertainty by } \\
\text { fuzzy soft intervals }\end{array}$ \\
\hline Zhang et al. [55] & IFS & $\checkmark$ & $\checkmark$ & $\checkmark$ & $\times$ & $\checkmark$ & $\begin{array}{l}\mathrm{D} \\
\text { us }\end{array}$ & $\begin{array}{l}\text { als uncertainty by } \\
\text { ing MD and NMD }\end{array}$ \\
\hline Xu et al. [56] & IFS & $\checkmark$ & $\checkmark$ & $\checkmark$ & $\times$ & $\times$ & $\begin{array}{l}\mathrm{D} \\
\text { us }\end{array}$ & $\begin{array}{l}\text { als uncertainty by } \\
\text { ing MD and NMD }\end{array}$ \\
\hline Maji et al. [9] & IFSS & $\checkmark$ & $\checkmark$ & $\checkmark$ & $x$ & $\times$ & $\begin{array}{l}\mathrm{D} \\
\text { us }\end{array}$ & $\begin{array}{l}\text { als uncertainty by } \\
\text { ing MD and NMD }\end{array}$ \\
\hline $\begin{array}{l}\text { Proposed } \\
\text { approach }\end{array}$ & IFHSS & $\checkmark$ & $\checkmark$ & $\checkmark$ & $\checkmark$ & $\times$ & $\begin{array}{l}\text { Dec } \\
\text { col }\end{array}$ & $\begin{array}{l}\text { ls more uncertainty } \\
\text { nparative to IFHSS }\end{array}$ \\
\hline
\end{tabular}

\subsection{Comparative Analysis}

We recommend another algorithmic rule under IFHSS by utilizing the established IFHSWA and IFHSWG operators in the following section. Subsequently, we utilize the suggested algorithm to a realistic problem, namely the supplier selection in SSCM. The overall outcomes prove that the algorithmic rule is valuable and practical. It can be observed that $\mathcal{Q}^{(4)}$ supplier is the finest alternative for SSCM (see Table 6). The recommended approach may be compared to other available methods. From the research findings, it has been concluded that the outcomes acquired by the planned approach exceed the consequences of the prevailing ideas. Therefore, comparative to existing techniques, the established AOs competently handled the uncertain and ambiguous information. However, under existing DM strategies, the core advantage of the planned method is that it can accommodate extra information in data comparative to existing techniques. It is also a beneficial tool to solve inaccurate, as well as imprecise, information in DM procedures.

Table 6. Comparative analysis with existing operators.

\begin{tabular}{cccccc}
\hline Method & $\mathcal{Q}^{(1)}$ & $\mathcal{Q}^{(2)}$ & $\mathcal{Q}^{(3)}$ & $\mathcal{Q}^{(4)}$ & Ranking Order \\
\hline IFWA [25] & 0.21173 & 0.22017 & 0.33215 & 0.27008 & $\mathcal{Q}^{(4)}>\mathcal{Q}^{(1)}>\mathcal{Q}^{(2)}>\mathcal{Q}^{(3)}$ \\
IFWG [21] & 0.20587 & 0.23066 & 0.32902 & 0.25462 & $\mathcal{Q}^{(4)}>\mathcal{Q}^{(1)}>\mathcal{Q}^{(2)}>\mathcal{Q}^{(3)}$ \\
IFEWA [22] & 0.51686 & 0.54833 & 0.60467 & 0.59021 & $\mathcal{Q}^{(4)}>\mathcal{Q}^{(1)}>\mathcal{Q}^{(2)}>\mathcal{Q}^{(3)}$ \\
IFEWG [20] & 0.54219 & 0.56597 & 0.62190 & 0.59381 & $\mathcal{Q}^{(4)}>\mathcal{Q}^{(1)}>\mathcal{Q}^{(2)}>\mathcal{Q}^{(3)}$ \\
IFSWA [13] & 0.08158 & 0.07674 & 0.14762 & 0.09959 & $\mathcal{Q}^{(4)}>\mathcal{Q}^{(1)}>\mathcal{Q}^{(2)}>\mathcal{Q}^{(3)}$ \\
IFSWG [13] & 0.49830 & 0.41735 & 0.40935 & 0.46175 & $\mathcal{Q}^{(4)}>\mathcal{Q}^{(1)}>\mathcal{Q}^{(2)}>\mathcal{Q}^{(3)}$ \\
Proposed IFHSWA operator & -0.195086 & -0.124363 & 0.084652 & 0.095501 & $\mathcal{Q}^{(4)}>\mathcal{Q}^{(3)}>\mathcal{Q}^{(2)}>\mathcal{Q}^{(1)}$ \\
Proposed IFHSWG operator & -0.259867 & -0.242376 & -0.141950 & -0.035913 & $\mathcal{Q}^{(4)}>\mathcal{Q}^{(3)}>\mathcal{Q}^{(2)}>\mathcal{Q}^{(1)}$ \\
\hline
\end{tabular}


The existing operators are unable to cope with parametrization and multi sub-attributes of the alternatives. However, our developed IFHSWA and IFHSWG operators competently handle the parametrization and multi sub-attributes of the considered attributes. Therefore, it is a suitable tool to merge inexact as well as hesitant information in the DM method.

\section{Conclusions}

This study focuses on IFHSS to resolve the problems of inadequate, indistinct, and discrepant information by considering the MD and NMD on the n-tuple sub-attributes of the considered attributes. This research puts forward the novel aggregation operators for IFHSS, such as IFHSWA and IFHSWG operators with their fundamental properties. Additionally, a DM approach has been developed by using IFHSWA or IFHSWG operators to solve MCDM complications. Furthermore, a comparative analysis was performed to ensure the effectiveness and manifestation of the presented approach. Lastly, according to the obtained outcomes, it can be determined that the planned method displays advanced constancy and feasibility for experts in the DM procedure. Based on the obtained results, it can be determined that the planned technique shows that the experts have higher stability and availability in the DM process. The forthcoming study will focus on presenting DM techniques utilizing numerous other measures, such as entropy measures and similarity measures, etc., under IFHSS. Moreover, many other structures can be established and proposed, such as topological structure, algebraic structure, ordered structure, etc. We believe that this research opens new vistas for investigators in this field.

Author Contributions: Conceptualization, R.M.Z.; methodology, R.M.Z.; validation, I.S. and D.P.; formal analysis, R.A. and D.M.; investigation, D.P. and D.M.; writing-original draft preparation, R.M.Z.; writing-review and editing, D.P. and D.B.; visualization, D.B.; supervision, I.S. and D.P.; funding acquisition, D.P., D.M. and D.B. All authors have read and agreed to the published version of the manuscript.

Funding: This research received no funding.

Institutional Review Board Statement: Not applicable.

Informed Consent Statement: Informed consent was obtained from all subjects involved in the study.

Data Availability Statement: Not applicable.

Acknowledgments: The author Rifaqat Ali would like to express his gratitude to Deanship of Scientific Research at King Khalid University, Saudi Arabia for providing funding research groups under the research grant number R. G. P. 1/162/42.

Conflicts of Interest: The authors declare no conflict of interest.

\section{References}

1. Zadeh, L.A. Fuzzy sets. Inf. Control 1965, 8, 338-353. [CrossRef]

2. Atanassov, K.T. Intuitionistic fuzzy sets. Fuzzy Sets Syst. 1986, 20, 87-96. [CrossRef]

3. Garg, H.; Kaur, G. Cubic intuitionistic fuzzy sets and its fundamental properties. J. Mult. Valued Logic Soft Comput. 2019, 33, 507-537.

4. Atanassov, K.; Gargov, G. Interval valued intuitionistic fuzzy sets. Fuzzy Sets Syst. 1989, 31, 343-349. [CrossRef]

5. Li, Y.Y. Entropy and Distance Measure of Intuitionistic Fuzzy Soft Sets. Adv. Mater. Res. 2014, 1070-1072, 2056-2061. [CrossRef]

6. Molodtsov, D. Soft Set Theory First Results. Comput. Math. Appl. 1999, 37, 19-31. [CrossRef]

7. Maji, P.K.; Biswas, R.; Roy, A.R. Fuzzy soft sets. J. Fuzzy Math. 2001, 9, 589-602.

8. Ali, G.; Akram, M.; Koam, A.N.A.; Alcantud, J.C.R. Parameter Reductions of Bipolar Fuzzy Soft Sets with Their Decision-Making Algorithms. Symmetry 2019, 11, 949. [CrossRef]

9. Maji, P.K.; Biswas, R.; Roy, A. Intuitionistic fuzzy soft sets. J. Fuzzy Math. 2001, 9, 677-692.

10. Garg, H.; Arora, R. TOPSIS method based on correlation coefficient for solving decision-making problems with intuitionistic fuzzy soft set information. AIMS Math. 2020, 5, 2944-2966. [CrossRef]

11. Wei, C.; Zhang, Y. Entropy Measures for Interval-Valued Intuitionistic Fuzzy Sets and Their Application in Group DecisionMaking. Math. Probl. Eng. 2015, 2015, 563745. [CrossRef] 
12. Zulqarnain, R.M.; Xin, X.L.; Saqlain, M.; Khan, W.A. TOPSIS Method Based on the Correlation Coefficient of Interval-Valued Intuitionistic Fuzzy Soft Sets and Aggregation Operators with Their Application in Decision-Making. J. Math. 2021, 2021, 6656858. [CrossRef]

13. Arora, R.; Garg, H. A robust aggregation operators for multi-criteria decision-making with intuitionistic fuzzy soft set environment. Sci. Iran. 2017, 25, 931-942. [CrossRef]

14. Wang, L.; Garg, H.; Li, N. Pythagorean fuzzy interactive Hamacher power aggregation operators for assessment of express service quality with entropy weight. Soft Comput. 2021, 25, 973-993. [CrossRef]

15. Wang, L.; Li, N. Continuous interval-valued Pythagorean fuzzy aggregation operators for multiple attribute group decision making. J. Intell. Fuzzy Syst. 2019, 36, 6245-6263. [CrossRef]

16. Wang, L.; Garg, H.; Li, N. Interval-valued q-rung orthopair 2-tuple linguistic aggregation operators and their applications to decision making process. IEEE Access 2019, 7, 131962-131977. [CrossRef]

17. Pamucar, D. Normalized weighted Geometric Dombi Bonferoni Mean Operator with interval grey numbers: Application in multicriteria decision making. Rep. Mech. Eng. 2020, 1, 44-52. [CrossRef]

18. Peng, X.; Yuan, H. Fundamental Properties of Pythagorean Fuzzy Aggregation Operators. Fundam. Inform. 2016, 147, 415-446. [CrossRef]

19. Garg, H. A New Generalized Pythagorean Fuzzy Information Aggregation Using Einstein Operations and Its Application to Decision Making. Int. J. Intell. Syst. 2016, 31, 886-920. [CrossRef]

20. Wang, W.; Liu, X. Intuitionistic fuzzy geometric aggregation operators based on einstein operations. Int. J. Intell. Syst. 2011, 26, 1049-1075. [CrossRef]

21. Xu, Z.; Yager, R.R. Some geometric aggregation operators based on intuitionistic fuzzy sets. Int. J. Gen. Syst. 2006, 35, 417-433. [CrossRef]

22. Wang, W.; Liu, X. Intuitionistic Fuzzy Information Aggregation Using Einstein Operations. IEEE Trans. Fuzzy Syst. 2012, 20, 923-938. [CrossRef]

23. Arora, R.; Garg, H. Group decision-making method based on prioritized linguistic intuitionistic fuzzy aggregation operators and its fundamental properties. Comput. Appl. Math. 2019, 38, 36. [CrossRef]

24. Faizi, S.; Sałabun, W.; Nawaz, S. Best-Worst method and Hamacher aggregation operations for intuitionistic 2-tuple linguistic sets. Expert Syst. Appl. 2021, 115088. [CrossRef]

25. Xu, Z. Intuitionistic fuzzy aggregation operators. IEEE Trans. Fuzzy Syst. 2007, 15, 1179-1187.

26. Sinani, F.; Erceg, Z.; Vasiljević, M. An evaluation of a third-party logistics provider: The application of the rough Dombi-Hamy mean operator. Decis. Mak. Appl. Manag. Eng. 2020, 3, 92-107.

27. Riaz, M.; Çagman, N.; Wali, N.; Mushtaq, A. Certain properties of soft multi-set topology with applications in multi-criteria decision making. Decis. Mak. Appl. Manag. Eng. 2020, 3, 70-96. [CrossRef]

28. Peng, X.; Yang, Y.; Song, J. Pythagoren fuzzy soft set and its application. Comput. Eng. 2015, 41, $224-229$.

29. Athira, T.M.; John, S.J.; Garg, H. A novel entropy measure of pythagorean fuzzy soft sets. AIMS Math. 2020, 5, 1050-1061. [CrossRef]

30. Zulqarnain, R.M.; Xin, X.L.; Garg, H.; Khan, W.A. Aggregation operators of Pythagorean fuzzy soft sets with their application for green supplier chain management. J. Intell. Fuzzy Syst. 2021, 40, 5545-5563. [CrossRef]

31. Riaz, M.; Sałabun, W.; Farid, H.M.A.; Ali, N.; Wątróbski, J. A Robust q-Rung Orthopair Fuzzy Information Aggregation Using Einstein Operations with Application to Sustainable Energy Planning Decision Management. Energies 2020, 13, 2155. [CrossRef]

32. Faizi, S.; Sałabun, W.; Ullah, S.; Rashid, T.; Więckowski, J. A New Method to Support Decision-Making in an Uncertain Environment Based on Normalized Interval-Valued Triangular Fuzzy Numbers and COMET Technique. Symmetry 2020, 12, 516. [CrossRef]

33. Bashir, Z.; Rashid, T.; Watróbski, J.; Sałabun, W.; Malik, A. Hesitant probabilistic multiplicative preference relations in group decision making. Appl. Sci. 2018, 8, 398. [CrossRef]

34. Kushwaha, D.K.; Panchal, D.; Sachdeva, A. Risk analysis of cutting system under intuitionistic fuzzy environment. Rep. Mech. Eng. 2020, 1, 162-173. [CrossRef]

35. Sałabun, W.; Watróbski, J.; Shekhovtsov, A. Are MCDA Methods Benchmarkable? A Comparative Study of TOPSIS, VIKOR, COPRAS, and Promethee II Methods. Symmetry 2020, 12, 1549. [CrossRef]

36. Smarandache, F. Extension of Soft Set to Hypersoft Set, and then to Plithogenic Hypersoft Set. Neutrosophic Sets Syst. 2018, 22, 168-170.

37. Rana, S.; Qayyum, M.; Saeed, M.; Smarandache, F. Plithogenic Fuzzy Whole Hypersoft Set: Construction of Operators and their Application in Frequency Matrix Multi Attribute Decision Making Technique. Neutrosophic Sets Syst. 2019, 28 , 34-50.

38. Zulqarnain, R.M.; Xin, X.L.; Saqlain, M.; Smarandache, F. Generalized Aggregate Operators on Neutrosophic Hypersoft Set. Neutrosophic Sets Syst. 2020, 36, 271-281.

39. Zulqarnain, R.M.; Xin, X.L.; Saeed, M. A Development of Pythagorean fuzzy hypersoft set with basic operations and decisionmaking approach based on the correlation coefficient. In Theory and Application of Hypersoft Set; Pons Publishing House Brussels: Brussels, Belgium, 2021; Chapter 5; pp. 85-106. 
40. Zulqarnain, R.M.; Siddique, I.; Jarad, F.; Ali, R.; Abdeljawad, T. Development of TOPSIS Technique under Pythagorean Fuzzy Hypersoft Environment Based on Correlation Coefficient and Its Application towards the Selection of Antivirus Mask in COVID-19 Pandemic. Complexity 2021, 2021, 6634991. [CrossRef]

41. Samad, A.; Zulqarnain, R.M.; Sermutlu, E.; Ali, R.; Siddique, I.; Jarad, F.; Abdeljawad, T. Selection of an Effective Hand Sanitizer to Reduce COVID-19 Effects and Extension of TOPSIS Technique Based on Correlation Coefficient Under Neutrosophic Hypersoft Set. Complexity 2021, 2021, 1-23.

42. Zulqarnain, R.M.; Xin, X.L.; Saqlain, M.; Saeed, M.; Smarandache, F.; Ahamad, M.I. Some Fundamental Operations on Interval Valued Neutrosophic Hypersoft Set with Their Properties. Neutrosophic Sets Syst. 2021, 40, 134-148.

43. Zulqarnain, R.M.; Xin, X.L.; Ali, B.; Broumi, S.; Abdal, S.; Ahamad, M.I. Decision-Making Approach Based on Correlation Coefficient with its Properties Under Interval-Valued Neutrosophic hypersoft set environment. Neutrosophic Sets Syst. 2021, 40, $12-28$.

44. Zulqarnain, R.M.; Xin, X.L.; Saeed, M. Extension of TOPSIS method under intuitionistic fuzzy hypersoft environment based on correlation coefficient and aggregation operators to solve decision making problem. AIMS Math. 2021, 6, 2732-2755. [CrossRef]

45. Chatterjee, P.; Stević, Ž. A two-phase fuzzy AHP-fuzzy TOPSIS model for supplier evaluation in manufacturing environment. Oper. Res. Eng. Sci. Theory Appl. 2019, 2, 72-90. [CrossRef]

46. Milenkov, M.A.; Sokolović, V.S.; Milovanović, V.R.; Milić, M.D. A role, significance and approaches for studying logistics. Vojn. Glas. 2020, 68, 79-106. [CrossRef]

47. Zavadskas, E.K.; Turskis, Z.; Stević, Ž.; Mardani, A. Modelling Procedure for the Selection of Steel Pipes Supplier by Applying Fuzzy AHP Method. Oper. Res. Eng. Sci. Theory Appl. 2020, 3, 39-53. [CrossRef]

48. Wątróbski, J.; Sałabun, W.; Ladorucki, G. The Temporal Supplier Evaluation Model Based on Multicriteria Decision Analysis Methods. In Proceedings of the Asian Conference on Intelligent Information and Database Systems, Kanazawa, Japan, 3-5 April 2017; Springer: Cham, Switzerland, 2017; pp. 432-442.

49. Vasiljevic, M.; Fazlollahtabar, H.; Stević, Ž.; Vesković, S. A Rough Multicriteria Approach for Evaluation of the Supplier Criteria in Automotive Industry. Decis. Mak. Appl. Manag. Eng. 2018, 1, 82-96. [CrossRef]

50. Wątróbski, J.; Sałabun, W. Green Supplier Selection Framework Based on Multi-Criteria Decision-Analysis Approach. In Proceedings of the International Conference on Sustainable Design and Manufacturing, Chania, Greece, 4-6 April 2016; Springer: Cham, Switzerland, 2016; pp. 361-371.

51. Todorov, T.; Mitrev, R.; Penev, I. Force analysis and kinematic optimizationof a fluid valve driven by shape memory alloys. Rep. Mech. Eng. 2020, 1, 61-76. [CrossRef]

52. Marković, D.; Petrovć, G.; Ćojbašić, Ž.; Stanković, A. The Vehicle Routing Problem with Stochastic Demands in an Urban Area-A Case Study. Facta Univ. Ser. Mech. Eng. 2020, 18, 107-120. [CrossRef]

53. Milošević, T.D.; Pamučar, D.S.; Chatterjee, P. Model for selecting a route for the transport of hazardous materials using a fuzzy logic system. Vojn. Glas. 2021, 69, 355-390. [CrossRef]

54. Zolfani, S.H.; Yazdani, M.; Pamucar, D.; Zarate, P. A Vikor and Topsis Focused Reanalysis of the Madm Methods Based on Logarithmic Normalization. Facta Univ. Ser. Mech. Eng. 2020, 18, 341-355. [CrossRef]

55. Zhang, H.M.; Xu, Z.S.; Chen, Q. On clustering approach to intuitionistic fuzzy sets. Contr. Decis. 2007, 22, 882-888.

56. Xu, Z.; Chen, J.; Wu, J. Clustering algorithm for intuitionistic fuzzy sets. Inf. Sci. 2008, 178, 3775-3790. [CrossRef] 